\title{
Development of Methods and Modeling of Individual Characteristics of the Target of Magnetic Inertial Synthesis under Combined Exposure
}

\author{
V.V. Kuzenov ${ }^{1,2}$, V.V.Shumaev ${ }^{2}$ \\ ${ }^{1}$ Dukhov Research Institute of Automatics (VNIIA), \\ Moscow, 127055, Russia \\ 2 Bauman Moscow State Technical University, \\ Moscow, 105005, Russia \\ vik.kuzenov@gmail.com; chubchic@gmail.com
}

\begin{abstract}
The work is devoted to the theoretical calculation of the physical and technical characteristics of the combined effect of intense energy fluxes on the target in the magnetic inertial plasma confinement method. The results of calculating the effect of intense energy flows on a singlelayer cylindrical target are presented. Based on these calculations, the possibility of creating compact neutron generators was evaluated. The new algorithm for numerically solving the "hyperbolic" and "parabolic" parts of the equations of plasma dynamics is briefly described.
\end{abstract}

Keywords: high-temperature plasma, magnetized target, mathematical modeling, development of numerical methods, plasmodynamic processes

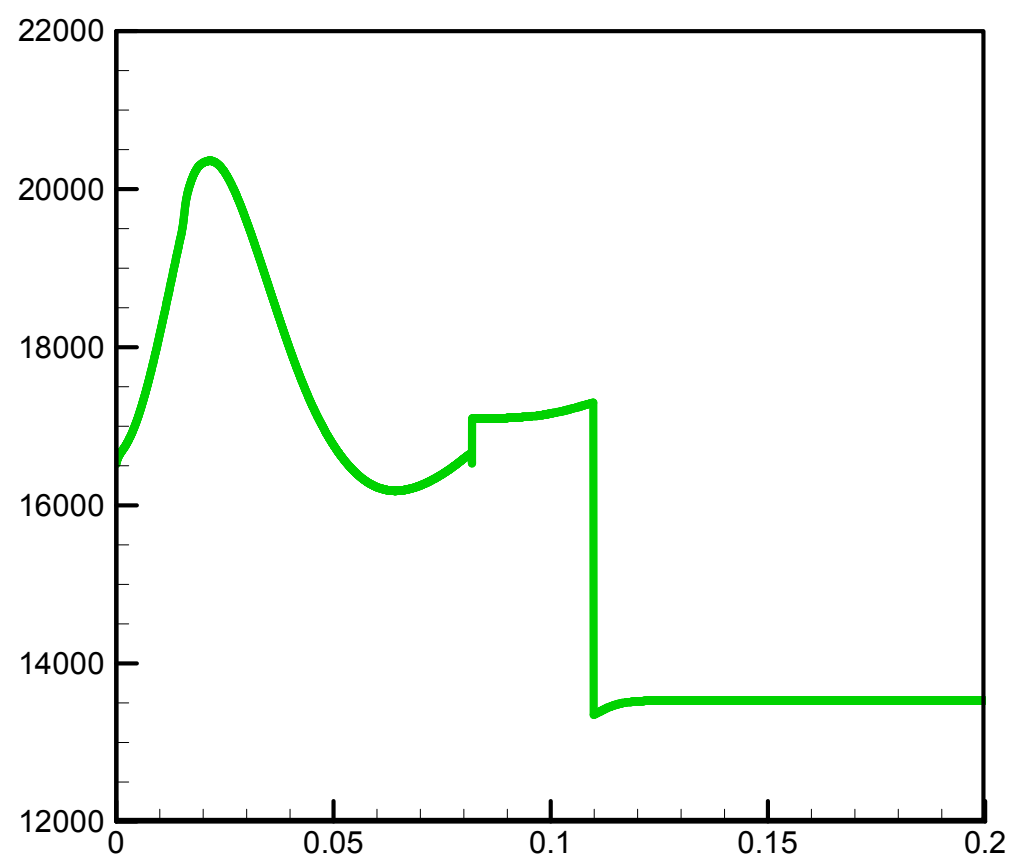

Spatial temperature distribution $\mathrm{T}[\mathrm{kK}]$ for $10^{14} \mathrm{~W} / \mathrm{cm}^{2}$, number of neutrons per unit length $3.08 \times 10^{14} \mathrm{n} / \mathrm{cm}$ for a time point of $9.99 \mathrm{~ns}$ 


\title{
Разработка методики и моделирование отдельных характеристик мишени магнитно- инерциального синтеза при комбинированном воздействии
}

\author{
В.В. Кузенов ${ }^{1,2}$, В.В. Шумаев ${ }^{2}$ \\ ${ }^{1}$ Федеральное государственное унитарное предприятие «Всероссийский научно- \\ исследовательский институт автоматики им. Н.Л. Духова», \\ Россия, Москва, 127055, ул. Сущёвская, д.22 \\ ${ }^{2}$ МГТУ им. Н.Э. Баумана, Россия, Москва, 105005, 2-я Бауманская ул., д. 5, стр. 1 \\ vik.kuzenov@gmail.com; chubchic@gmail.com
}

\begin{abstract}
Аннотация
Работа посвящена расчетно-теоретической оценке физико-технических характеристик комбинированного способа воздействия интенсивных потоков энергии на мишень в магнитно-инерционном способе удержания плазмы. Представлены результаты расчета воздействия интенсивных потоков энергии на однослойную цилиндрическую мишень. На основе этих расчетов оценена возможность создания компактных генераторов нейтронов. Кратко описан новый алгоритм численного решения «гиперболической» и «параболической» части уравнений плазмодинамики.
\end{abstract}

Ключевые слова: высокотемпературная плазма, замагниченная мишень, математическое моделирование, разработка численных методов, плазмодинамические процессы.

\section{1. Введение}

Магнитно-инерциальный термоядерный синтез (МИТС) [1-8] основывается на возбуждении термоядерных реакций в плазменном образовании центральной части мишени, состоящей из дейтерия (D) и трития (T), с помощью имплозии стенки мишени лазерными пучками с высокой энергией импульса (лазерным драйвером) и/или высокоскоростными плазменными струями (плазменным драйвером). Здесь отметим, что первым существенным отличием «плазменного драйвера» от лазерного драйвера является то, что в этом случае внешняя граница сжимаемой мишени образуется системой ударных волн, которая ограничивает, в отличие от лазерного драйвера (в этом случае здесь располагается волна разряжения - плазменная корона), радиальное расширение плазмы мишени МИТС. Второе важное отличие связано с тем обстоятельством, что трансформация направленной кинетической энергии плазменных струй во внутреннюю энергию материала мишени более эффективно в сравнении с лазерным воздействием т.к. осуществляется за счет обмена энергией между «тяжелыми» частицами, а не путем менее эффективного взаимодействия квантов лазерного излучения с электронами мишени.

Процесс нагрева качественно может быть описан следующим образом: после прохождения через стенку (сопровождающееся необратимым нагреванием материала стенки) мишени сильной ударной волны (при условии, что внешнее подведенное давление превышает тепловое давление материала стенки мишени МИТС, а скорость тепловой или ионизационной волны ниже скорости ударной волны (УВ)), ускорения стенки мишени и генерации за счет этого ударной волны в термоядерной плазме мишени, кумуляции их на геометриче- 
ской оси системы приводит к сжатию и нагреву (до стадии протекания термоядерных реакций) плазмы мишени.

В полной постановке (многомерной, многоскоростной, многотемпературной среды с учетом многих возможных физико-химических процессов - первый уровень вычислительной модели [11]) это чрезвычайно сложная задача даже для современных высокопроизводительных вычислительных комплексов. Математическая модель этой задачи формулируется на основе системы уравнений для моментов функций распределения частиц по скоростям, уравнений Максвелла, переноса широкополосного излучения и «реальных» уравнений состояния.

Однако многие задачи физики магнитно-инерциального термоядерного синтеза могут быть относительно хорошо исследованы с помощью МГД моделей. Однако и в этом случае, в силу сложности рассматриваемой задачи, необходимо сделать ряд упрощающих предположений (второй уровень вычислительной модели [11]), среди которых выделим следующие основные:

- $\quad$ будем считать, что плазменное образование (состав плазмы одноатомный) состоит из диссоциированного нейтрального и диссоциированного ионизованного газа. Математическое описание динамики такого плазменного образования основывается на многожидкостной (трехскоростной), многотемпературной, химически нереагирующей сплошной плазменной среды;

- $\quad$ расчет динамических процессов (поглощение и рассеяние), связанных с решением кинетического уравнения Власова (или Фоккера-Планка) для релятивистских «надтепловых» электронов в магнитном поле, не рассматривается;

- $\quad$ в начальный момент времени плазменное образование, и окружающая среда разделены заданием различных термодинамических характеристик и степенью ионизации $\alpha=n_{e} / n$, где $n_{e}, n-$ концентрации электронов и «тяжелых» частиц.

К третьему уровню вычислительной модели [11] можно отнести математическую модель (1)-(7) процессов сжатия и энерговыделения в мишени МИТС (1D модель), приведенную ниже по тексту. Она позволяет оценить эволюцию сжатия и энерговыделения в мишени МИТС при последовательном (относительно времени) увеличении интенсивности системы ударных волн, падающих на геометрическую ось системы, благодаря комбинированному воздействию на неё интенсивного лазерного излучения (в торцевом - на завершающей стадии сжатия и перпендикулярном образующей направлениях) и внешней (по отношению к мишени), ограничивающей расширение плазмы мишени, системы импульсных струй.

Одной из целей данной работы является оценка возможности создания компактных генераторов нейтронов на основе комбинированной схемы воздействия. В принципе такая схема может позволить сформировать нейтронный выход на уровне $10^{12} \div 10^{16}$ нейтронов за импульс.

\section{2. Математическая модель}

Математическая модель процессов сжатия и энерговыделения в мишени МИТС, представленная в данной работе, опирается на одномерные уравнения радиационной плазмодинамики (1)-(7), записанные в центрально-симметричной системе координат: систему уравнений Эйлера (1), локального (термоядерного) энерговыделения (2), уравнение переноса лазерного (3) и собственного широкополосного излучения (7), уравнения магнитной индукции (5),(6), методы расчета уравнений состояний вещества и коэффициентов поглощения лазерного излучения (4)

$$
\frac{\partial \rho}{\partial t}+\frac{\partial(\rho u)}{\partial r}=F_{\rho}, F_{\rho}=-\rho u \frac{(v-1)}{r}
$$




$$
\begin{gathered}
\frac{\partial(\rho u)}{\partial t}+\frac{\partial\left(\rho u^{2}+P\right)}{\partial r}=F_{\rho u}+f_{r}, \quad F_{\rho u}=-\left(\rho u^{2}-P\right) \frac{(v-1)}{r}, \quad f_{r}=\frac{1}{c}[\vec{j} \times \vec{H}]_{r}, \\
\frac{\partial(\rho E)}{\partial t}+\frac{\partial\left(\rho E u+P u+q_{\Sigma}\right)}{\partial r}=F_{E}+q_{r}+Q_{F u s}^{e}, \quad q_{r}=j_{r} E_{r}+j_{z} E_{z}, \quad q_{\Sigma}=q_{e}+q_{i}+q_{l a z}, \\
F_{E}=-(\rho E u+P u) \frac{(v-1)}{r}, P=P_{e}+P_{i}, \quad q_{b u t t}(r)=\frac{2 q_{t}}{\delta} \exp \left[-\chi_{\omega}(r) \delta\right],
\end{gathered}
$$

где $t$ - время; $r$ - радиальная координата; $\rho$ - плотность; $u$ - скорость вдоль координаты $r$; $P=P(\rho, e)-$ статическое давление; $e-$ удельная внутренняя энергия; $E=\left(e+u^{2} / 2\right)-$ полная энергия потока газа; $\vec{F}=\left(F_{\rho}, F_{\rho u}, F_{E}\right)$ - вектор источников в центрально-симметричной системе координат; $F_{\rho}$ - плотность потока массы; $F_{\rho u}$ - плотность потока импульса; $F_{E}-$ плотность потока энергии; $q_{v}, q=\sum_{v} q_{v}-$ спектральный и полный поток широкополосного излучения; $T_{e}, T_{i}$ - температуры электронов и ионов плазмы $\left(T=T_{e}=T_{i}\right) ; f_{r}$ - электромагнитная сила; $q_{r}-$ приток энергии от электромагнитного поля; $q_{e}=-\lambda_{e} \operatorname{grad} T_{e}, q_{i}=-\lambda_{i} \operatorname{grad} T_{i}$; $\lambda_{e}, \lambda_{i}-$ коэффициенты теплопроводности электронов и ионов; $j_{r}$ - плотность тока; $\vec{H}(r)-$ вектор магнитной индукции; $P_{e}$ - давление электронов; $P_{i}$ - давление ионов; $q_{b u t t}(r), q_{l a z}-$ плотность потока лазерного излучение соответственно в торцевом (в направлении оси $z$ ) направлении и перпендикулярно (в направлении оси $r$ ) образующей цилиндрической мишени; $\delta$ - длина мишени МИТС в направлении оси $z$ ( $\delta=0.2$ см); индекс $v=(1,2,3)$ - отвечает случаям плоской, осевой и сферической симметрии.

В качестве граничных условий системы уравнений Эйлера (1) ставится условие симметрии (на оси симметрии), на внешней границе задаются не возмущающие условия на выходящий из расчетной области поток: $\partial^{2} \vec{f} /\left.\partial r^{2}\right|_{r=\ell}=0$, где $\vec{f}=\{\rho, u, e\}$.

Вклад локального (термоядерного) энерговыделения $Q_{F u s}^{e}(2)$ в электронный компонент плазмы за счет передачи энергии электронам от термоядерных $\alpha$ - частиц может быть определен с помощью приближенной формулы [9]

$$
Q_{\text {Fus }}^{e}(r, t)=8.483 \times 10^{29} \rho^{2} \frac{\left(1+0.232 \times T_{i}^{3 / 4}\right) \exp \left(-20 / T_{i}^{1 / 3}\right)}{T_{i}^{2 / 3} \sqrt{1+9.41 \times 10^{-5} \times T_{i}^{13 / 4}}}\left[Э \mathrm{p} /\left(\mathrm{cm}^{3} \cdot \mathrm{c}\right)\right],
$$

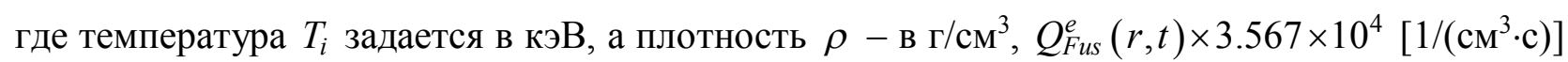
- число нейтронов, образующихся за секунду в единице объема.

Параметры лазерного излучения вдоль оси $r$ находятся на основе решения уравнения переноса лазерного излучения (3)

$$
\frac{\mathrm{d} q_{l a z}}{\mathrm{~d} z}-\chi_{\omega} q_{l a z}=0
$$

При этом коэффициент поглощения $\chi_{\omega}\left(\mathrm{cm}^{-1}\right)$ лазерного излучения определяется с использованием механизма континуального поглощения, обратного механизму тормозного излучения электронов в условиях локального термодинамического равновесия (ЛТР)

$$
\chi_{\omega}= \begin{cases}\frac{4.97 g Z_{i}^{2} n_{i}^{\Sigma} n_{e}^{\Sigma}}{n_{c}^{2} \lambda^{2}\left(k T_{e}\right)^{3 / 2}} \frac{1}{\sqrt{1-n_{e} / n_{c}}}, & n_{e}<n_{c} \\ \infty, & n_{e} \geq n_{c}\end{cases}
$$


где $\lambda$ - длина волны излучения лазера (мкм); $n_{e}, n_{i}-$ концентрации электронов и ионов $\left(\mathrm{cm}^{3}\right)$; $k T_{e}$ - электронная температура в (кэВ); $g$ - фактор Гаунта [36]; $\rho_{c}=1.865 \times 10^{-3} \frac{A_{i}}{Z_{i}} \times \lambda(\mu \mathrm{m})^{-2}$, $\left[г / \mathrm{cm}^{3}\right]$ - критическая плотность электронов (здесь $A_{i}=M_{i} / m_{p}-$ атомный номер иона, $M_{i}-$ атомная масса иона, $m_{P}=1.67262158 \times 10^{-24}$, [г] - масса протона). Излучение, доходящее до точки, в которой $\rho=\rho_{c}$, полностью поглощается в окрестности этой точки.

Расчет лазерного излучения вдоль оси $r$ должен сопровождаться соответствующими граничными условиями: $\left.q_{l a z}\right|_{r=\ell}$ (сформулированы ниже по тексту), $\left.q_{l a z}\right|_{r=0}=0$.

Отметим, что источником спонтанного магнитного поля $\vec{B}$ с компонентами $B_{z}$ или $B_{\varphi}$ при воздействии интенсивных потоков энергии на стенки мишени МИТС может являться неколлинеарность $\left(\nabla n_{e} \times \nabla T \neq 0\right)$ градиентов плотности электронов $\nabla n_{e}$ и температуры $\nabla T$. Заметим также, что если указанная неоднородность присутствует при изменении азимутального угла $\varphi$, то спонтанное магнитное поле $\vec{B}$ для рассматриваемой формы мишени имеет компоненту $B_{z}$.

Оценка модуля вектора $|\vec{B}|$ спонтанного магнитного поля основана на следующем выражении [3]:

$$
\left|\frac{\partial \vec{B}}{\partial t}\right| \approx\left|\frac{c k}{e n_{e}}\left[\nabla n_{e} \times \nabla T\right]\right| \approx \frac{c k}{e} \frac{T_{x}}{r_{l a z}^{2}}
$$

При характерных значениях масштабов $r_{l a z}, T_{x}, q_{l a z}^{0}, t_{x}$ характерная величина магнитного поля будет иметь значение $|\vec{B}| \geq 1$ МГс. Здесь $r_{l a z} \approx 10^{-2} \mathrm{~cm}-$ характерный размер плазменного образования вдоль радиальной координаты; $T_{x} \approx 1 \times 10^{6} \mathrm{~K}$ - характерное значение температуры плазмы; $q_{\text {laz }}^{0} \approx 10^{14} \mathrm{BT} / \mathrm{cm}^{2}$ - характерное значение плотности потока лазерного излучения; $t_{x} \approx 10$ нс - характерное временя воздействия лазерного излучения.

Уравнение магнитной индукции (5) для компоненты поля $B_{\varphi}$, учитывающее уравнение неразрывности для плотности $\rho$, закон сохранения вида $\operatorname{div}(\vec{B})=0$ и источник спонтанного магнитного поля, можно записать в следующем виде [10,11]:

$$
\begin{gathered}
\frac{\partial\left(B_{\varphi} / \rho\right)}{\partial t}+\frac{1}{\mu J r} \frac{\partial J r\left(u B_{\varphi} / \rho\right)}{\partial r}=\frac{c^{2}}{4 \pi \mu \rho J r} \frac{\partial}{\partial r}\left(\frac{J}{\sigma} \frac{\partial r B_{\varphi}}{\partial r}\right)-\frac{c k}{\rho e}\left[\nabla n_{e} \times \nabla T\right]_{\varphi}, \frac{\partial r B_{\varphi}}{r \partial r}=j_{z}, \\
{\left[\nabla \ln \left(n_{e}\right) \times \nabla T\right]_{\varphi}=\left(\frac{\partial \ln \left(n_{e}\right)}{\partial r} \frac{\partial T}{\partial z}-\frac{\partial \ln \left(n_{e}\right)}{\partial z} \frac{\partial T}{\partial r}\right)}
\end{gathered}
$$

В соотношения (5) входят производные $\partial T / \partial z, \partial \ln \left(n_{e}\right) / \partial z$ (в принципе делающие решаемую задачу - $2 D$ задачей), рассчитываемые вдоль образующей цилиндрической мишени МИТС. В данной работе (с целью упрощения постановки задачи) эти производные определялись приближенно из предположения выполнимости условия линейного (т.е. $\partial T / \partial z \approx$ const и $\partial \ln \left(n_{e}\right) / \partial z \approx$ const ) изменения величин $T$ и $\ln \left(n_{e}\right)$ вдоль координаты $z$.

Уравнение магнитной индукции (6) для компоненты поля $B_{z}$ (внешнее магнитное поле) записывается следующим образом $[10,11]$ :

$$
\frac{\partial\left(B_{z} / \rho\right)}{\partial t}+\frac{1}{\mu J} \frac{\partial J\left(u B_{z} / \rho\right)}{\partial r}=\frac{c^{2}}{4 \pi \mu \rho J r} \frac{\partial}{\partial r}\left(\frac{J r}{\sigma} \frac{\partial B_{z}}{\partial r}\right), \frac{\partial r B_{z}}{r \partial r}=j_{\varphi}
$$

Электропроводность $\sigma$, входящая в уравнения (5) и (6), определяется по формуле Спитцера [12] с учетом возможной замагниченности плазмы 


$$
\sigma=12.06 \times 10^{13} \cdot \frac{T^{3 / 2}}{\ln \Lambda} \cdot \frac{n_{e}}{\sum_{i} n_{i} z_{i}^{2}} \frac{1}{1+\left(\Omega_{e} \tau_{e}\right)^{2}},\left(\frac{1}{\text { сек }}\right)
$$

где $z_{i}, n_{i}$ - средний заряд и концентрация ионов; $\Omega_{e}=e|\vec{B}| / m_{e} c-$ гирочастота электронов; $m_{e}-$ масса электрона; $\tau_{e}-$ среднее время электрон-ионных столкновений с передачей импульса.

Краевые условия необходимые при решении уравнений магнитной индукции (5) и (6) можно описать следующим образом: на оси симметрии - условие симметрии, на внешней границе $-\partial \vec{H}(r) / \partial r=0$.

Перенос широкополосного излучения (7) рассматривается с помощью многогруппового диффузионного приближения, уравнения которого выглядят следующим образом [5]:

$$
\frac{1}{r^{n}} \frac{\mathrm{d}\left(r^{n} q_{v}\right)}{\mathrm{d} r}+\chi_{v} c U_{v}=\chi_{v} 4 \sigma T^{4}, \quad \frac{c}{3} \frac{\mathrm{d} U_{v}}{\mathrm{~d} r}+\chi_{v} q_{v}=0,
$$

где $q_{v}, U_{v}$ - спектральный поток и объемная плотность широкополосного излучения; $c$ скорость света; $v$ - номер частотной группы, $\chi_{v}-$ спектральный коэффициент поглощения; $n=0$ - плоский слой; $n=1$ - бесконечный одномерный цилиндр; $n=2$ - сферически симметричный случай. Здесь под величиной $q_{v}$ понимается радиационный поток в направлении оси $r$.

Граничные условия для системы уравнений диффузионного приближения (7) могут быть сформулированы следующим образом: на внешней границе - отсутствие падающего извне излучения $\left.q_{v}\right|_{r=r_{n}}=\frac{c}{2} U_{v}$, на оси симметрии - условие симметрии

$$
\left.q_{v}\right|_{r=0}= \begin{cases}0, & n=1,2 \\ -\frac{c}{2} U_{v}, & n=0\end{cases}
$$

Отметим, что обеспечить энергетически выгодный режим изоэнтропического сжатия вещества центральной части мишени МИТС достаточно сложно: временная форма лазерного импульса $\left.q_{l a z}(t)\right|_{r=\ell}$ должна быть спрофилирована специальным образом, длительность импульса согласована с радиусом и массой оболочки мишени МИТС. Однако в этом случае в принципе имеется возможность получения заметных степеней сжатия $c: c=\rho_{f} / \rho_{i}$ где $\rho_{i}$ и $\rho_{f}$ - начальное и конечное значения плотности центральной части вещества мишени МИТС, но при этом конечная температура сжатия $T_{f}$ относительно невелика. Здесь важно отметить, что для практической реализации МИТС нужен высокой уровень температур: $T_{f} \approx 10^{8} \mathrm{~K}$. Существенным (перестает соблюдаться соотношение $T / \rho^{\gamma-1}=$ const, $\gamma=$ const) является также, то, что в процессе сжатия заметно изменяется (по сравнению началом процесса) «эффективный» показатель адиабаты $\gamma$ и в этом случае необходимо применять реальное уравнение состояния. Таким образом, для достижения данного уровня конечных температур $T_{f}$ за времена много меньше времени пробега звуковой волны по несжатому газу сжатие мишени МИТС следует начинать с помощью системы сильных УВ [14]. Поэтому в работе была применена концепция hot-spot. В этом случае реализуется ситуация, когда вещество мишени МИТС сжимается в первой серии ударных волн, а термоядерный нагрев (поджиг) осуществляется в последней ударной волне большой амплитуды. Для этой указанной цели можно использовать профилированный (по специальному закону) временной импульс плотности потока лазерного излучения $\left.q_{l a z}(t)\right|_{r=\ell}$. При этом параметры, падающего (вдоль координаты $r$ ) на мишень МИТС лазерного излучения, могут находиться путем 
решения уравнения переноса лазерного излучения $[13,15]$ с учетом соответствующих граничных условий:

$$
\left.q_{l a z}\right|_{r=\ell}=\left\{\begin{array}{ll}
q_{\text {laz }}^{0}\left(t / t_{0}\right)^{n}, & t \leq t_{0} \\
0, & t_{0}<t<t_{0}+\Delta t
\end{array},\right.
$$

где $\frac{\left.\int_{0}^{t} q_{\text {laz }}(t)\right|_{r=\ell} \mathrm{d} t}{\left.\int_{0}^{t_{0}} q_{\text {laz }}(t)\right|_{r=\ell} \mathrm{d} t}=0.5-$ функциональное условие для нахождения времени обострения импульса $t_{\varepsilon}=\frac{t_{0}}{2^{1 /(n+1)}}=7.17 \mathrm{нс}$.

Обычно используется еще и дополнительное условие вида

$$
\frac{t_{0}-t_{\varepsilon}}{t_{0}} \ll 1
$$

$q_{\text {laz }}^{0}=1 \times 10^{14} \mathrm{BT} / \mathrm{cm}^{2} ; n=2.8 ; t_{0}=8.6 \mathrm{Hc} ; \Delta t=1.48 \mathrm{Hc}$.

Поток лазерного излучения, поступающего на торцевую часть мишени, находится следующим способом:

$$
q_{t}=\left\{\begin{array}{ll}
0, & t \leq t_{0}, \\
q_{l a z}^{1}, & t_{0}<t<t_{0}+\Delta t,
\end{array}, q_{l a z}^{1}=1 \times 10^{15} \mathrm{BT} / \mathrm{cm}^{2}\right.
$$

Значение плотности потока энергии $q$ на границе расчетной области в импульсной струе задается выражением

$$
\left.q\right|_{r=\ell}=\left\{\begin{array}{ll}
0, & t \leq t_{0}, \\
q^{0}, & t_{0}<t<t_{0}+\Delta t
\end{array}, \text { где } q^{0} \in\left[10^{11}, 10^{12}\right] \text { Вт } / \mathrm{cm}^{2}\right.
$$

Возможность создания импульсных струй плазмы с указанными параметрами следует из результатов работы [15].

Коэффициенты электронной и ионной теплопроводности $\lambda_{e, i}$ в случае замагниченной плазмы могут быть рассчитаны с помощью формул [12]. Расчет, входящих в данную систему уравнений термодинамических $e(T, \rho), P(T, \rho)$ и оптических параметров рабочих сред, проводился в рамках приближения ЛТР с использованием компьютерной системы ASTEROID, разработанной академиком РAH C.T. Суржиковым [17], модели Томаса-Ферми с квантовыми и обменными поправками $[18,19]$ и модели среднего заряда [11].

Начальное значения напряженности $\vec{H}(r)$ «затравочного» магнитного поля в разреженной окружающей среде составляет доли Тл. Спектральный поток и объемная плотность широкополосного излучения $q_{v}, U_{v}$, а также лазерный поток излучения $q_{l a z}$ для $r \in[0, \ell]$ в начальный момент времени $t=0$ равны нулю.

\section{3. Численный метод расчета}

Метод численного решения одномерных уравнений плазмодинамики мишеней МИТС опирается на метод дробных шагов и описан в работе [13]. Несмотря на одномерный характер задачи, она предъявляет повышенные требования к численному методу, используемому при её решении. Прежде всего, расчетная схема должна обладать улучшенными дисперсионными и диссипативными свойствами, быть экономичной и алгоритмически простой, об- 
ладать свойством монотонности и аппроксимировать гладкие решения желательно с максимально высоким порядком точности. Этим требованиям удовлетворяет метод численного решения однотемпературных, одножидкостных уравнений магнитной газовой динамики, который опирается на метод дробных шагов, который состоит в данном случае из двух шагов [20].

На первом дробном шаге учитываются газодинамические процессы (этим процессам соответствует «гиперболическая» часть рассматриваемой системы уравнений). При этом процессы переноса излучения и электромагнитные процессы, протекающие в устройствах системы МИТС, рассматриваются на втором дробном шаге.

При численном решении такого рода задач часто применяются подвижные сетки, адаптирующиеся к особенностям численного решения. Такой подход позволяет получать результаты повышенной точности на относительно грубых расчетных сетках. Использование динамически адаптивных сеток приводит к необходимости осуществить переход от осесимметричной системы координат $r, t$ к произвольным подвижным (в 2D и $3 \mathrm{D}$ случаях к криволинейным) $\xi, t$ координатам.

Поэтому в дальнейшем математическая формулировка первого дробного шага и решение «гиперболической» части системы уравнений (1) использует систему координат $\xi, t$ и основывается на дивергентной форме вида (8)

$$
\frac{\partial \vec{U}}{\partial t}+\frac{\partial F(\vec{U})}{\partial \xi}=\vec{F}_{2} \text {, или } \frac{\partial \vec{U}}{\partial t}=L(U), L=-\frac{\partial F(\vec{U})}{\partial \xi}+\vec{F}_{2}
$$

Здесь вектор решения $\vec{U}$ и вектор потоковой переменной $F(\vec{U})$ имеют вид

$$
\vec{U}=\left(\rho, \rho u_{\xi}, \rho E\right)^{T}, F(\vec{U})=\left(\rho u_{\xi}, \rho u_{\xi}^{2}+P, \rho E u_{\xi}+P u_{\xi}\right)^{T}
$$

Вектор правой части уравнений (8) представляется следующим образом

$$
\vec{F}_{2}=\left(F_{\rho}, F_{\rho u}, F_{E}\right)^{T}
$$

Отметим, что выше приведенные система дифференциальных уравнений (8) относительно временной переменной $t$ есть система обыкновенных дифференциальных уравнений (ОДУ) первого порядка, которые могут быть разрешены с помощью векторного варианта многошагового метода Рунге-Кутта (в данной работе использован четырех шаговый вариант метода [21], который обладает 4-м порядком аппроксимации по времени $t$ ).

Для этого приведем векторный вариант системы уравнений Эйлера (8) к нормальной форме с выделенной в левой части временной производной $\frac{\partial \vec{U}_{i}}{\partial t}$

$$
\frac{\partial \vec{U}_{i}}{\partial t}=L\left(\vec{U}_{i}\right),
$$

где $L$ - правая часть системы уравнений Эйлера не содержащая производных по времени. В качестве начального приближения используется решение, полученное на предыдущем шаге по времени. Тогда четырехшаговый вариант метода Рунге-Кутта реализуется в виде следующей последовательности шагов:

$$
\begin{array}{cc}
\vec{U}_{i}^{(1)}=\left[\vec{U}_{i}^{(0)}+\frac{\Delta t}{4} L\left(\vec{U}_{i}^{(0)}\right)\right], & \vec{U}_{i}^{(2)}=\left[\vec{U}_{i}^{(0)}+\frac{\Delta t}{3} L\left(\vec{U}_{i}^{(1)}\right)\right], \\
\vec{U}_{i}^{(3)}=\left[\vec{U}_{i}^{(0)}+\frac{\Delta t}{2} L\left(\vec{U}_{i}^{(2)}\right)\right], & \vec{U}_{i}^{(4)}=\left[\vec{U}_{i}^{(0)}+\Delta t L\left(\vec{U}_{i}^{(3)}\right)\right]
\end{array}
$$


Известно, что такой способ поиска решения $\vec{U}_{i}$ относительно времени $t$ позволяет решить одну из проблем численного решения уравнений Эйлера - необходимость обеспечить положительность искомых функций (если в момент времени $t^{n}$ решение является положительным, то оно остается положительным и в момент времени $t^{n+1}$ ).

Повышение относительно временной переменной $t$ порядка аппроксимации численного решения уравнений Эйлера до четвертого $O\left(\Delta t^{4}\right)$ и выше также возможно, если использовать последовательность сеток по временной переменной $t$ и экстраполяцию по пределу, предложенную Ричардсоном. Экстраполяция Ричардсона имеет следующие особенности:

- возможность использования простейших аппроксимаций дифференциальных задач;

- $\quad$ однородность осуществления алгоритмов на последовательности сеток с различными параметрами аппроксимации;

- $\quad$ простота реализации алгоритма в целом.

Пусть имеется решение, рассматриваемой задачи найденное интегрированием (со вторым порядком аппроксимации $O\left(\Delta t^{2}\right)$ ) по времени $t$ с шагом $\Delta t$ на момент времени $\hat{t}=t+\Delta t$, которое обозначим $\left.(\rho, u, P)\right|_{t+\Delta t}$, а также решение (имеющее также второй порядок аппроксимации $O\left(\Delta t^{2}\right)$, обозначаемое, как $F_{i}=\frac{\partial}{\partial \xi}+\frac{\Delta_{\xi}^{8}}{44100}+O\left(\Delta_{\xi}^{10}\right)$, полученное с использованием двух временных шагов (величина каждого шага равна $\Delta t / 2$ ) до момента времени $\hat{t}=t+\Delta t$.

Тогда линейная комбинация вида $\overline{\overline{\left.(\rho, u, P)\right|_{t+\Delta t} ^{\hat{t}}}}=\left.\frac{4}{3}(\rho, u, P)\right|_{t+\Delta t / 2} ^{\hat{t}}-\left.\frac{1}{3}(\rho, u, P)\right|_{t+\Delta t} ^{\hat{t}}$ приближает точное решение с четвертым порядком аппроксимации по временной переменной $O\left(\Delta t^{4}\right)$ [22]. Чтобы точное решение можно было приблизить с шестым или восьмым порядком аппроксимации по временной переменной, следует воспользоваться формулами вида [23]

$$
\begin{aligned}
& \overline{\overline{\left.(\rho, u, P)\right|_{t+\Delta t} ^{\hat{t}}}}=\left.\frac{32}{21}(\rho, u, P)\right|_{t+\Delta t / 4} ^{\hat{t}}-\left.\frac{4}{7}(\rho, u, P)\right|_{t+\Delta t / 2} ^{\hat{t}}+\left.\frac{1}{21}(\rho, u, P)\right|_{t+\Delta t} ^{\hat{t}}, \\
& \overline{\overline{\left.(\rho, u, P)\right|_{t+\Delta t} ^{\hat{t}}}}=\left.\frac{512}{315}(\rho, u, P)\right|_{t+\Delta t / 8} ^{\hat{t}}-\left.\frac{32}{45}(\rho, u, P)\right|_{t+\Delta t / 4} ^{\hat{t}}+\left.\frac{4}{45}(\rho, u, P)\right|_{t+\Delta t / 2} ^{\hat{t}}-\left.\frac{1}{315}(\rho, u, P)\right|_{t+\Delta t} ^{\hat{t}}
\end{aligned}
$$

Как уже отмечалось выше, на первом дробном шаге используется дивергентная форма уравнений Эйлера в системе координат $\xi, t$

$$
\frac{\partial \rho}{\partial t}+\frac{\partial \rho u_{\xi}}{\partial \xi}=F_{\rho}, \quad \frac{\partial\left(\rho u_{\xi}\right)}{\partial t}+\frac{\partial\left(\rho u_{\xi}^{2}+P\right)}{\partial \xi}=F_{\rho u}, \quad \frac{\partial(\rho E)}{\partial t}+\frac{\partial\left(\rho E u_{\xi}+P u_{\xi}\right)}{\partial \xi}=F_{E}
$$

Данную систему уравнений можно записать в векторной форме

$$
\frac{\partial \vec{U}}{\partial t}+\frac{\partial F(\vec{U})}{\partial \xi}=\vec{F}_{2}
$$

где $u_{\xi}=(u)$, вектор решения имеет вид $\vec{U}=\left(\rho, \rho u_{\xi}, \rho E\right)^{T}$, вектор потоковой переменной записывается в виде $F(\vec{U})=\left(\rho u_{\xi}, \rho u_{\xi}^{2}+P, \rho E u_{\xi}+P u_{\xi}\right)^{T}$, а вектор правой части представляется следующим образом: $\vec{F}_{2}=\left(F_{\rho}, F_{\rho u}, F_{E}\right)^{T}$. Здесь (для временного дробного шага 
$t \in[t, t+\Delta t / 2])$ применяется нелинейная квазимонотонная компактно-полиномиальная разностная схема повышенного порядка точности

$$
\frac{\partial \vec{U}_{i}}{\partial t}+\frac{F\left(\vec{U}_{i+1 / 2}\right)-F\left(\vec{U}_{i-1 / 2}\right)}{\Delta_{\xi}}=\vec{F}_{2}, \quad \Delta_{\xi}=\left[\xi_{i-1 / 2}-\xi_{i}, \xi_{i+1 / 2}-\xi_{i}\right]
$$

В этом соотношении газодинамические параметры $U_{i}^{n+1}, U_{i}^{n}$ относятся к центрам расчетных ячеек, в то время как потоки $F_{i \pm 1 / 2}^{n}$ необходимо определить на поверхности этих ячеек. Для повышения порядка аппроксимации разностной схемы следует «восстановить» газодинамические параметры $Y_{i \pm 1 / 2}^{R, L}$ «справа» (индекс $-R$ ) и «слева» (индекс $\left.-L\right)$ от границ расчетных ячеек. Тогда любая реконструируемая функция $Y(\xi), \xi \in\left[-\frac{\Delta_{\xi}}{2}, \frac{\Delta_{\xi}}{2}\right]$, представляется кусочно-полиномиальными распределениями вида

$$
Y(\xi)=F_{i}^{n}(\xi)=R(\xi)+a_{i}\left[\xi-\xi_{i}\right]^{3}+b_{i}\left[\xi-\xi_{i}\right]^{4}+c_{i}\left[\xi-\xi_{i}\right]^{5}+d_{i}\left[\xi-\xi_{i}\right]^{6}+e_{i}\left[\xi-\xi_{i}\right]^{7}
$$

Главная часть $R(\xi)$ «реконструируемой» функции $Y(\xi)$ на момент времени $t^{n}$ определяется выражением вида

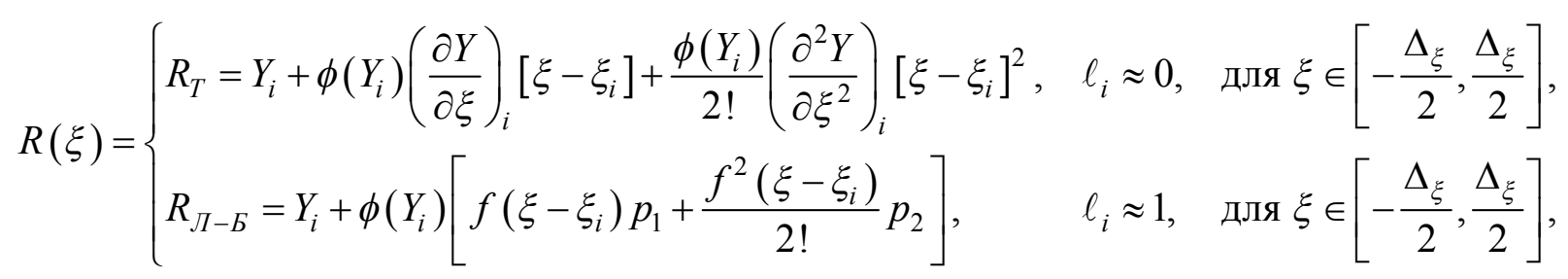

где $p_{1}, p_{2}$ - первые коэффициенты разложения функции $Y(\xi)$ в усеченный ряд Лагранжа-Бюрмана определены ниже по тексту. Очевидно, что точность аппроксимации (с помощью главной части $R(\xi))$ «реконструируемой» функции $Y(\xi)$ зависит от порядка точности (ошибки аппроксимации) восстановления значений производных $\left(\frac{\partial Y}{\partial \xi}\right)_{i}$ и $\left(\frac{\partial^{2} Y}{\partial \xi^{2}}\right)_{i}$.

Из выражения главной части $R(\xi)$, соответствующей «реконструируемой» функции следует, что для кусочно-гладких (или гладких) функций $Y(\xi)$, возможно содержащих «слабые» разрывы $\left(\ell_{i} \approx 0, \operatorname{Ind}(Y) \approx 1\right)$, главная часть $R(\xi)=R_{T}$ «реконструируемой» функции на момент времени $t^{n}$ основывается на ряде Тейлора. А для функций $Y(\xi)$, содержащих «сильные» разрывы $\left(\ell_{i} \approx 1, \operatorname{Ind}(Y) \approx 1\right)$, при описании главной части $R(\xi)=R_{J-5}$ «реконструируемой» функции используется разложение в ряд Лагранжа-Бюрмана по степеням некоторой функции $f(\xi)$, аппроксимирующей ступенчатую (разрывную) функцию Хевисайда.

Данные кусочно-полиномиальные распределения следует ограничить (для придания им монотонного вида) некоторой функцией $\phi(Y)$ - лимитером, который имеет следующий вид [24]:

$$
\phi\left(Y_{i}\right)=\min \left(1, \frac{\left|Y_{i}-\max \left(Y_{k}\right)\right|}{\left|Y_{i}-\max \left(Y_{k-1 / 2}, Y_{k+1 / 2}\right)\right|}, \frac{\left|Y_{i}-\min \left(Y_{k}\right)\right|}{\left|Y_{i}-\min \left(Y_{k-1 / 2}, Y_{k+1 / 2}\right)\right|}\right)
$$

Функция $Y(x)$ удовлетворяет условиям гладкого сопряжения

$$
F_{i}^{n}\left(\xi_{i-1}\right)=Y_{i-1}^{n}, \quad F_{i}^{n}\left(\xi_{i+1}\right)=Y_{i+1}^{n}, \frac{\mathrm{d} F_{i}^{n}\left(\xi_{i-1}\right)}{\mathrm{d} \xi}=Y_{\xi, i-1}^{n}, \frac{\mathrm{d} F_{i}^{n}\left(\xi_{i+1}\right)}{\mathrm{d} \xi}=Y_{\xi, i+1}^{n},
$$


и условию консервативности реконструируемой функции $Y(x)$

$$
\frac{1}{\Delta_{\xi}} \int_{-\frac{\Delta_{\xi}}{2}}^{+\frac{\Delta_{\xi}}{2}} Y_{i}^{n}(\xi) \mathrm{d} \xi=Y\left(\xi_{i}\right)
$$

Указанные выше условия гладкого сопряжения можно сформулировать в виде системы линейных алгебраических уравнений

$$
\begin{aligned}
& A \overrightarrow{Z_{i}}=\vec{F}_{i}, \vec{Z}_{i}=\left(a_{i}, b_{i}, c_{i}, d_{i}, e_{i}\right)^{\mathrm{T}}, \overrightarrow{F_{i}}=\left(F_{1}, F_{2}, F_{3}, F_{4}, F_{5}\right)^{\mathrm{T}}, \\
& A=\left(\begin{array}{ccccc}
-\Delta_{\xi}{ }^{3} & \Delta_{\xi}{ }^{4} & -\Delta_{\xi}{ }^{5} & \Delta_{\xi}{ }^{6} & -\Delta_{\xi}{ }^{7} \\
3 \Delta_{\xi}{ }^{2} & -4 \Delta_{\xi}{ }^{3} & 5 \Delta_{\xi}{ }^{4} & -6 \Delta_{\xi}{ }^{5} & 7 \Delta_{\xi}{ }^{6} \\
\Delta_{\xi}{ }^{3} & \Delta_{\xi}{ }^{4} & \Delta_{\xi}{ }^{5} & \Delta_{\xi}{ }^{6} & \Delta_{\xi}{ }^{7} \\
3 \Delta_{\xi}{ }^{2} & 4 \Delta_{\xi}{ }^{3} & 5 \Delta_{\xi}{ }^{4} & 6 \Delta_{\xi}{ }^{5} & 7 \Delta_{\xi}{ }^{6} \\
0 & \frac{1}{5}\left(\frac{\Delta_{\xi}}{2}\right)^{4} & 0 & \frac{1}{7}\left(\frac{\Delta_{\xi}}{2}\right)^{6} & 0
\end{array}\right), \\
& F_{1}=Y_{i-1}^{n}-Y_{i}+\phi\left(Y_{i}\right)\left(\frac{\partial Y}{\partial \xi}\right)_{i} \Delta_{\xi}-\frac{\phi\left(Y_{i}\right)}{2 !}\left(\frac{\partial^{2} Y}{\partial \xi^{2}}\right)_{i} \Delta_{\xi}^{2}, \quad F_{2}=Y_{\xi, i-1}^{n}-\phi\left(Y_{i}\right)\left(\frac{\partial Y}{\partial \xi}\right)_{i}+\phi\left(Y_{i}\right)\left(\frac{\partial^{2} Y}{\partial \xi^{2}}\right)_{i} \Delta_{\xi}, \\
& F_{3}=Y_{i+1}^{n}-Y_{i}-\phi\left(Y_{i}\right)\left(\frac{\partial Y}{\partial \xi}\right)_{i} \Delta_{\xi}-\frac{\phi\left(Y_{i}\right)}{2 !}\left(\frac{\partial^{2} Y}{\partial \xi^{2}}\right)_{i} \Delta_{\xi}^{2}, \quad F_{4}=Y_{\xi, i+1}^{n}-\phi\left(Y_{i}\right)\left(\frac{\partial Y}{\partial \xi}\right)_{i}-\phi\left(Y_{i}\right)\left(\frac{\partial^{2} Y}{\partial \xi^{2}}\right)_{i} \Delta_{\xi}, \\
& F_{5}=-\frac{\phi\left(Y_{i}\right)}{3 !}\left(\frac{\partial^{2} Y}{\partial \xi^{2}}\right)_{i}\left[\frac{\Delta_{\xi}}{2}\right]^{2}
\end{aligned}
$$

В данных условиях матрица $A$ фиксирована, а значит, фиксирована и обратная матрица, $A^{-1}$ т.е. она может быть найдена до проведения основных расчетов. При этом коэффициенты $Z_{i}=\left(a_{i}, b_{i}, c_{i}, d_{i}, e_{i}\right)^{\mathrm{T}} i$-го полинома можно найти из очевидного соотношения $Z_{i}=A^{-1} F_{i}$.

Входящие в кусочно-полиномиальные распределения $Y(\xi)$ пространственные производные $\left(\frac{\partial Y}{\partial \xi}\right)_{i, j}$, вычисляются следующим образом: сначала для дискретной функции $Y_{i}$ определим приближенное значение $F_{i}$ первой частной производной по пространственной переменной $\xi$ с восьмым порядком точности. Для этого в каждой ячейке с номером $i$ для каждой восстанавливаемой величины $Y_{i, j}$ осуществляется расчет индекса немонотонности $\operatorname{Ind}(Y)$

$$
\operatorname{Ind}(Y)_{i}=\frac{\frac{1}{12}\left|-Y_{i+2, j}+16 Y_{i+1, j}-30 Y_{i, j}+16 Y_{i-1, j}-Y_{i-2, j}\right|}{\frac{1}{2}\left|-Y_{i+2, j}+4 Y_{i+1, j}-3 Y_{i, j}\right|+\frac{1}{2}\left|3 Y_{i, j}-4 Y_{i-1, j}+Y_{i-2, j}\right|+\theta},
$$

где величина $\theta$ является малым параметром. 
Далее найдем первую производную $f_{i}$ по переменной $\xi$ по обычной аппроксимационной формуле второго порядка точности и произведем её «монотонное ограничение» на сетке

$$
\tilde{f}_{i}=\phi\left(Y_{i}\right) f_{i}, \quad f_{i}=\frac{Y_{i+1}-Y_{i-1}}{2 \Delta_{\xi}}+O\left(\Delta_{\xi}^{2}\right)
$$

где $\Delta$ - шаг пространственной сетки в направлении $\xi$. Тогда приближенное «монотонизованное» значение $\tilde{F}_{i}$ первой частной производной по пространственным переменным $\xi \mathrm{c}$ ошибкой аппроксимации на уровне $F_{i}=\frac{\partial}{\partial \xi}+\frac{\Delta_{\xi}^{6}}{2100}+O\left(\Delta_{\xi}^{8}\right)$ можно найти по формуле вида (т.е. путем решения системы уравнений с трехдиагональной матрицей)

$$
Q_{i}=\left(E+\frac{\Delta_{2}}{30}\right) \tilde{f}_{i}, \quad \widetilde{F}_{i}=\left\{\left(E+\frac{\Delta_{2}}{6}\right)^{-1} Q_{i}\right\}_{i},
$$

где $\Delta_{0} f_{i}=f_{i+1}-f_{i-1}, \Delta_{2} f_{i}=f_{i+1}-2 f_{i}+f_{i-1} ; M$ - единичный оператор. Данная формула является симметричной конечной разностью шестого порядка точности [25].

$$
Q_{i}=\left(E+\frac{5 \Delta_{2}}{42}\right) \tilde{f}_{i}, \quad F_{i}=\left\{\left(E+\frac{2 \Delta_{2}}{7}+\frac{\Delta_{2}^{2}}{70}\right)^{-1} Q_{i}\right\}_{i}
$$

В кусочно-полиномиальных распределениях $Y(\xi)$ присутствуют пространственные производные второго порядка $\left(\frac{\partial^{2} Y}{\partial \xi^{2}}\right)_{i}=s_{i}$, которые обозначаются символом $s_{i}$ и вычисляются с восьмым порядком точности [23]

$$
\frac{9}{38}\left(s_{i+1}+s_{i-1}\right)+s_{i}=-\frac{751}{342 \Delta^{2}} Y_{i}+\frac{147}{152 \Delta^{2}}\left(Y_{i+1}+Y_{i-1}\right)+\frac{51}{380 \Delta^{2}}\left(Y_{i+2}+Y_{i-2}\right)-\frac{23}{6840 \Delta^{2}}\left(Y_{i+3}+Y_{i-3}\right)
$$

Далее с помощью реконструируемой функции $Y(\xi)$ «восстанавливаются» газодинамические параметры $Y_{i \pm 1 / 2}^{R, L}$ «справа» (индекс $-R$ ) и «слева» (индекс $-L$ ) от границ расчетных ячеек. Затем проводится «антидиффузионная» коррекция «восстанавливаемых» параметров $Y(\xi)$ на краях ячейки $Y_{i \pm 1 / 2}^{R, L}$.

«Антидиффузионная» коррекция параметров $Y_{i \pm 1 / 2}^{R, L}, Y_{i}^{R, L}$ проводится на основе следующего подхода [26-28]:

$$
\begin{aligned}
& \hat{Y}_{i+1 / 2}^{L}=Y_{i+1 / 2}^{L}+f_{*}\left(Y_{i}\right) \min \bmod \left(\frac{Y_{i}-Y_{i-1}}{\left(\max _{i}\left[V_{i}+c_{s, i}\right] \frac{\Delta t}{\Delta \xi}\right)}+Y_{i-1 / 2}^{L}-Y_{i+1 / 2}^{L}, Y_{i+1 / 2}^{R}-Y_{i+1 / 2}^{L}\right), \\
& \hat{Y}_{i-1 / 2}^{R}=Y_{i-1 / 2}^{R}-f_{*}\left(Y_{i}\right) \min \bmod \left(\frac{Y_{i}-Y_{i-1}}{\left(\max _{i}\left[V_{i}+c_{s, i}\right] \frac{\Delta t}{\Delta \xi}\right)}+Y_{i-1 / 2}^{L}-Y_{i+1 / 2}^{L}, Y_{i+1 / 2}^{R}-Y_{i+1 / 2}^{L}\right),
\end{aligned}
$$


где $V_{i}, c_{s, i}$ - скорость среды и скорость звука в каждой локальной точке $\xi_{i}$ распределения $Y(\xi)$. Функция $\min \bmod ($ ) определяется следующим образом:

$$
\min \bmod (a, b)=\left\{\begin{array}{lll}
0, & \text { если } a b \leq 0 \\
a, & \text { если } a b>0, & |a| \leq|b| \\
b, & \text { если } a b>0, & |b|>|a|
\end{array}\right.
$$

Индикатор разрыва $\ell_{i}$ меняется в диапазоне значений от 0 до $1\left(0 \leq f_{i} \leq 1\right)$ и находится следующим образом:

$$
\ell_{i}=\frac{\beta_{i}}{\beta_{i}+\gamma_{i}}
$$

где $\beta_{i}=\left[\frac{\alpha_{i}}{\alpha_{i-1}}+\frac{\alpha_{i+1}}{\alpha_{i+2}}\right]^{2}, \gamma_{i}=\frac{\left|Y_{\max }-Y_{\min }\right|^{2}}{\alpha_{i}}, Y_{\max }=\max _{i}\left[Y_{i}\right], Y_{\min }=\min _{i}\left[Y_{i}\right], \alpha_{i}=\left(\left|Y_{i-1}-Y_{i}\right|+\varepsilon\right)^{2}$, $\varepsilon \approx 10^{-6}$.

При этом функция индикатор разрыва $\ell\left(Y_{i}\right)$ модифицируется следующим образом:

$$
f_{*}\left(Y_{i}\right)=\frac{\ell\left(Y_{i}\right)}{c \ell\left(Y_{i}\right)+d\left[1-\ell\left(Y_{i}\right)\right]}\left[1-\operatorname{Ind}\left(Y_{i}\right)\right]^{0.25},
$$

где коэффициенты $c=2, d=2.25$. Отметим, что индикатор разрыва $\ell_{i}$ в гладкой области изменения функции $Y(\xi)$ имеет порядок $\ell_{i}=O\left(\Delta r^{2}\right)$ и близок к 1 вблизи сильных разрывов. Целью антидиффузионной поправки $\ell_{i}$ является улучшение разрешения контактных разрывов без ущерба точности и устойчивости исходной компактно-полиномиальной разностной схемы.

Из теории аппроксимации функций $Y(\xi)$ усеченным рядом Тейлора (некоторым многочленом) известно [29], что в окрестности разрывов исходной функции $Y(\xi)$ (и в областях больших градиентов решения) возникают осцилляции интерполирируемой функции. Однако функцию $Y(\xi)$ можно разложить в ряд более общего вида (в ряд Лагранжа-Бюрмана $[30,31])$ по степеням некоторой функции $f\left(\xi-\xi_{i}\right)$. При этом имеется возможность подобрать функцию $f(\xi)$ так, чтобы уменьшить амплитуду паразитических осцилляций численного решения в области $\ell_{i} \approx 1$. Пусть главная часть $R(\xi)$ реконструируемой функции $Y(\xi), \xi \in\left[-\frac{\Delta_{\xi}}{2}, \frac{\Delta_{\xi}}{2}\right]$, представлена в виде ряда по степеням функции $f(\xi)$ с помощью разложения Лагранжа-Бюрмана $[32,33]$, тогда реконструируемая функция $Y(\xi)$ может быть записана в виде

$$
\begin{gathered}
Y(\xi)=F_{i}^{n}(\xi)=Y_{i}+\phi\left(Y_{i}\right)\left\{f\left(\xi-\xi_{i}\right) p_{1}+f^{2}\left(\xi-\xi_{i}\right) p_{2} / 2 !+\right. \\
\left.+a_{i}\left[\xi-\xi_{i}\right]^{3}+b_{i}\left[\xi-\xi_{i}\right]^{4}+c_{i}\left[\xi-\xi_{i}\right]^{5}+d_{i}\left[\xi-\xi_{i}\right]^{6}+e_{i}\left[\xi-\xi_{i}\right]^{7}\right\}, \\
p_{1}=\frac{\left(\frac{\partial Y}{\partial \xi}\right)_{i}}{\left(\frac{\partial f}{\partial \xi}\right)_{i}}, \quad p_{2}=\frac{\left(\frac{\partial^{2} Y}{\partial \xi^{2}}\right)_{i}}{2\left(\frac{\partial f}{\partial \xi}\right)_{i}^{2}}-\frac{\left(\frac{\partial Y}{\partial \xi}\right)_{i}\left(\frac{\partial^{2} f}{\partial \xi^{2}}\right)_{i}}{2\left(\frac{\partial f}{\partial \xi}\right)_{i}^{3}}
\end{gathered}
$$


где $p_{1}, p_{2}$ - первые коэффициенты разложения функции $Y(\xi)$ в усеченный ряд Лагранжа-Бюрмана $[30,31]$. Отметим $[26,27]$, что при условии $f(\xi)=\xi$ коэффициенты $p_{1}, p_{2}$ переходят в коэффициенты обычного ряда Тейлора при соответствующих степенях монома $\left(\xi-\xi_{i}\right)$.

Здесь заметим, что в области разрыва (где индикатор разрыва $\ell_{i} \approx 1$ ) разложение в ряд Лагранжа-Бюрмана осуществляется по степеням монотонной функции $f(\xi)$, аппроксимирующей ступенчатую (разрывную) функцию Хевисайда

$$
f(\xi)=\Delta_{\xi} \operatorname{th}\left[\frac{\beta}{\Delta_{\xi}}\left(\xi-\xi_{i}\right)\right], \quad \beta=4 \cdot \operatorname{Ind}(Y)+6 \cdot[1-\operatorname{Ind}(Y)], \quad\left(\frac{\partial f}{\partial \xi}\right)_{i} \neq 0
$$

Для реконструируемой функции $Y(\xi)$ в области $\ell_{i} \approx 1$ (вблизи сильных разрывов) условия гладкого сопряжения и консервативности можно сформулировать в виде системы линейных алгебраических уравнений

$$
\begin{aligned}
& A \overrightarrow{Z_{i}}=\vec{F}_{i}, \quad \overrightarrow{Z_{i}}=\left(a_{i}, b_{i}, c_{i}, d_{i}, e_{i}\right)^{\mathrm{T}}, \quad \vec{F}_{i}=\left(F_{1}, F_{2}, F_{3}, F_{4}, F_{5}\right)^{\mathrm{T}}, \\
& A=\left(\begin{array}{ccccc}
-\Delta_{\xi}{ }^{3} & \Delta_{\xi}{ }^{4} & -\Delta_{\xi}{ }^{5} & \Delta_{\xi}{ }^{6} & -\Delta_{\xi}{ }^{7} \\
3 \Delta_{\xi}{ }^{2} & -4 \Delta_{\xi}{ }^{3} & 5 \Delta_{\xi}{ }^{4} & -6 \Delta_{\xi}{ }^{5} & 7 \Delta_{\xi}{ }^{6} \\
\Delta_{\xi}{ }^{3} & \Delta_{\xi}{ }^{4} & \Delta_{\xi}{ }^{5} & \Delta_{\xi}{ }^{6} & \Delta_{\xi}{ }^{7} \\
3 \Delta_{\xi}{ }^{2} & 4 \Delta_{\xi}{ }^{3} & 5 \Delta_{\xi}{ }^{4} & 6 \Delta_{\xi}{ }^{5} & 7 \Delta_{\xi}{ }^{6} \\
0 & \frac{1}{5}\left(\frac{\Delta_{\xi}}{2}\right)^{4} & 0 & \frac{1}{7}\left(\frac{\Delta_{\xi}}{2}\right)^{6} & 0
\end{array}\right), \\
& F_{1}=Y_{i-1}^{n}-Y_{i}-\phi\left(Y_{i}\right)\left\{-f\left(\Delta_{\xi}\right) p_{1}+\frac{f^{2}\left(\Delta_{\xi}\right)}{2 !} p_{2}\right\}, \quad F_{2}=Y_{\xi, i-1}^{n}-\phi\left(Y_{i}\right)\left\{p_{1}-f\left(\Delta_{\xi}\right) p_{2}\right\}\left(\frac{\partial f}{\partial \xi}\right)_{\Delta_{\xi}}, \\
& F_{3}=Y_{i+1}^{n}-Y_{i}-\phi\left(Y_{i}\right)\left\{f\left(\Delta_{\xi}\right) p_{1}+\frac{f^{2}\left(\Delta_{\xi}\right)}{2 !} p_{2}\right\}, \quad F_{4}=Y_{\xi, i+1}^{n}-\phi\left(Y_{i}\right)\left\{p_{1}+f\left(\Delta_{\xi}\right) p_{2}\right\}\left(\frac{\partial f}{\partial \xi}\right)_{\Delta_{\xi}} \text {, } \\
& F_{5}=-2 \phi\left(Y_{i}\right) p_{2}\left[1-\frac{2}{\beta} \operatorname{th}\left(\frac{\beta}{2}\right)\right]\left[\frac{\Delta_{\xi}}{2}\right]^{2}, \quad f(\xi)=\Delta_{\xi} \operatorname{th}\left[\frac{\beta}{\Delta_{\xi}}\left(\xi-\xi_{i}\right)\right]
\end{aligned}
$$

Таким образом, для нелинейной квазимонотонной компактно-полиномиальной разностной схемы повышенного порядка точности характерны следующие структурные особенности:

- $\quad$ предложенный способ «восстановления» распределения $Y(\xi)$ предполагает, что возможные разрывы решения сосредоточены на границах расчетной ячейки $q_{i}$;

- «восстанавливаемое» внутри расчетной ячейки $\xi \in\left[-\frac{\Delta_{\xi}}{2}, \frac{\Delta_{\xi}}{2}\right]$, распределение $Y(\xi)$ газодинамических параметров опирается на полином 7-й степени;

- $\quad$ главная часть $R(\xi)$ полинома (первые два слагаемых) определяется отдельным способом. При этом вблизи разрывов первого рода на основе разложения Лагранжа-Бюрмана по монотонным функциям; 
- $\quad$ первые $\left(\frac{\partial Y}{\partial \xi}\right)_{i}$ и вторые $\left(\frac{\partial^{2} Y}{\partial \xi^{2}}\right)_{i}$ производные находятся с использованием компактных разностей восьмого порядка точности, и значит главная часть $R(\xi)$ «реконструируемой» функции $Y(\xi)$ имеет такой же порядок аппроксимации;

- $\quad$ значения первых производных $\left(\frac{\partial Y}{\partial \xi}\right)_{i}$ монотонизируются с помощью специально введенного индекса немонотонности $\operatorname{Ind}(Y)$.

Процессы переноса излучения и электромагнитные процессы, протекающие в устройствах системы МИТС, рассматриваются на втором дробном шаге.

В этом случае для расчета тепловых процессов используется одномерное уравнение вида

$$
\tilde{c}(T) \frac{\partial T}{\partial t}=\frac{\partial}{\partial \xi}\left(\tilde{k}(T) \frac{\partial T}{\partial \xi}\right)+f(\xi, t)
$$

Для численного нахождения напряженностей магнитного поля можно (в общем случае) рассмотреть уравнение вида

$$
\frac{\partial H}{\partial t}+\frac{1}{\mu J} \frac{\partial J(u H)}{\partial \xi}=\frac{c^{2}}{4 \pi \mu J} \frac{\partial}{\partial \xi}\left(\frac{J}{\sigma} \frac{\partial H}{\partial \xi}\right), \quad J=\xi^{(v-1)}
$$

или уравнение вида

$$
\frac{\partial}{\partial t}\left(\frac{H_{z}}{\rho}\right)+u_{\xi} \frac{\partial}{\partial \xi}\left(\frac{H_{z}}{\rho}\right)=\frac{c^{2}}{4 \pi \rho J \xi} \frac{\partial}{\partial \xi}\left(\frac{J \xi}{\sigma} \frac{\partial H_{z}}{\partial \xi}\right)
$$

При этом для «гиперболической» части данных уравнений используется выше описанная нелинейная квазимонотонная разностная схема повышенного порядка точности. Тогда (в соответствии с методом расщепления по физическим процессам) необходимо описать лишь численный метод решения «параболической» части уравнений (уравнения диффузии).

$$
C \frac{\partial H}{\partial t} \approx \frac{\partial}{\partial \xi}\left(k \frac{\partial H}{\partial \xi}\right), \quad C=\frac{4 \pi \mu J}{c^{2}}, \quad k=\frac{J}{\sigma}
$$

Для построения численного решения уравнения диффузии магнитного поля и уравнения теплопроводности с помощью монотонизированной разностной схемы повышенного порядка точности заменим их эквивалентной системой двух уравнений первого порядка

$$
\begin{gathered}
C(\xi, t) \frac{\partial H}{\partial t}=-\frac{\partial w}{\partial \xi}, \quad \frac{\partial H}{\partial \xi}=-\frac{1}{k(\xi, t)} w(\xi, t), \\
H(\xi, t=0)=\varphi_{0}(\xi), \quad H(\xi=a, t)=\varphi_{a}(t), \quad H(\xi=b, t)=\varphi_{b}(t)
\end{gathered}
$$

Для численного построения решения введем сетку $\omega_{\tau} \times \omega_{h}: \omega_{h}=\left\{x_{i}=i \Delta \xi, i=\overline{0, N}\right\}$, $\omega_{\tau}=\left\{t_{n}=n \Delta t, i=0,1, \ldots\right\}$, (здесь $\Delta t$ и $\Delta \xi$ шаги по временной и пространственной переменным). Представим систему уравнений на сетке $\omega_{\tau} \times \omega_{h}$ в следующей конечно-разностной форме:

$$
-C\left(\xi_{i+1 / 2}, t_{n}\right) \frac{H^{n+1}-H^{n}}{\Delta t}=\frac{\partial w^{n+1}}{\partial \xi}, \quad \frac{\partial H^{n+1}}{\partial \xi}+\frac{1}{k\left(\xi_{i+1 / 2}, t_{n}\right)} w^{n+1}=0
$$


Тогда данную систему уравнений относительно функций $w$

$$
h^{n+1}=H^{n+1} \frac{k\left(\xi_{i+1 / 2}, t_{n}\right)}{\sqrt{a\left(\xi_{i+1 / 2}, t_{n}\right) \Delta t}},
$$

считая $k\left(\xi_{i+1 / 2}, t_{n}\right)$ и $C\left(\xi_{i+1 / 2}, t_{n}\right)$ постоянными, можно записать в следующем виде:

$$
\begin{gathered}
\frac{\partial w^{n+1}}{\partial \xi}+\frac{1}{\sqrt{a\left(\xi_{i+1 / 2}, t_{n}\right) \Delta t}} h^{n+1}=\frac{1}{\sqrt{a\left(\xi_{i+1 / 2}, t_{n}\right) \Delta t}} h^{n}, \\
\frac{\partial h^{n+1}}{\partial \xi}+\frac{1}{\sqrt{a\left(\xi_{i+1 / 2}, t_{n}\right) \Delta t}} w^{n+1}=0, a_{i+1 / 2, n}=\frac{k\left(\xi_{i+1 / 2}, t_{n}\right)}{C\left(\xi_{i+1 / 2}, t_{n}\right)}, \quad \xi \in\left[\xi_{i}, \xi_{i+1}\right]
\end{gathered}
$$

Будем искать решение в пределах расчетной ячейки $\left[\xi_{i}, \xi_{i+1}\right]$ в следующей форме:

$$
\begin{gathered}
w^{n+1}(\zeta)=C_{1} \exp \left(\frac{\zeta}{\sqrt{a_{i+1 / 2, n} \Delta t}}\right)+C_{2} \exp \left(-\frac{\zeta}{\sqrt{a_{i+1 / 2, n} \Delta t}}\right), \\
h^{n+1}(\zeta)=-\left(C_{1} \exp \left(\frac{\zeta}{\sqrt{a_{i+1 / 2, n} \Delta t}}\right)-C_{2} \exp \left(-\frac{\zeta}{\sqrt{a_{i+1 / 2, n} \Delta t}}\right)\right)+h^{n}(\zeta),
\end{gathered}
$$

где $\zeta=\xi-\xi_{i}, \zeta \in\left[0, \Delta \zeta=\xi_{i+1}-\xi_{i}\right]$.

Константы $C_{1}, C_{2}$ можно найти (здесь предполагается непрерывность функций $w$ и $H$ ) из граничных условий для потока $w:\left.w\right|_{\zeta=0}=w_{i},\left.w\right|_{\Delta \zeta=\xi_{i+1}-\xi_{i}}=w_{i+1}$ и функции $H:\left.H\right|_{\zeta=0}=H_{i}$, $\left.H\right|_{\Delta \zeta=\xi_{i+1}-\xi_{i}}=H_{i+1}$. Приравнивая попарно [5], получающиеся выражения для коэффициентов $C_{1}, C_{2}$, получим систему алгебраических связей между функциями $w(\xi)$ и $h(\xi)$

$$
\begin{gathered}
w_{i+1}^{n+1}-w_{i}^{n+1}+\operatorname{th}\left[\frac{\left(\xi_{i+1}-\xi_{i}\right) / 2}{\sqrt{a_{i+1 / 2} \Delta t}}\right]\left(h_{i}^{n+1}+h_{i+1}^{n+1}\right)=2 \operatorname{th}\left[\frac{\left(\xi_{i+1}-\xi_{i}\right) / 2}{\sqrt{a_{i+1 / 2} \Delta t}}\right] h_{i}^{n}, \\
\left(h_{i+1}^{n+1}-h_{i}^{n+1}\right)+2 \operatorname{th}\left[\frac{\left(\xi_{i+1}-\xi_{i}\right) / 2}{\sqrt{a_{i+1 / 2} \Delta t}}\right]\left(w_{i}^{n+1}+w_{i+1}^{n+1}\right)=0
\end{gathered}
$$

Здесь в проведенных рассуждениях принято, что величины $h$ и $w$ заданы в узловых точках $\xi_{i}$.

Запишем на отрезках $\left[\xi_{i-1 / 2}, \xi_{i}\right]$ и $\left[\xi_{i}, \xi_{i+1 / 2}\right]$ последнее уравнение системы алгебраических связей между функциями $W(\xi), h(\xi)$ следующим способом:

$$
\begin{aligned}
& h_{i}^{n+1}-h_{i-1 / 2}^{n+1}+2 \operatorname{th}\left[\frac{\left(\xi_{i}-\xi_{i-1 / 2}\right) / 2}{\sqrt{a_{i-1} \Delta t}}\right]\left(w_{i}^{n+1}+w_{i-1 / 2}^{n+1}\right)=0, \quad \xi \in\left[\xi_{i-1 / 2}, \xi_{i}\right] ; \\
& h_{i+1 / 2}^{n+1}-h_{i}^{n+1}+2 \operatorname{th}\left[\frac{\left(\xi_{i+1 / 2}-\xi_{i}\right) / 2}{\sqrt{a_{i} \Delta t}}\right]\left(w_{i+1 / 2}^{n+1}+w_{i}^{n+1}\right)=0, \quad \xi \in\left[\xi_{i}, \xi_{i+1 / 2}\right]
\end{aligned}
$$

Предполагая справедливым соотношение $w_{i}^{n+1} \approx w_{i-1 / 2}^{n+1} \approx w_{i+1 / 2}^{n+1}$, выражение для потока $w\left(\xi_{i}, t^{n+1}\right)$ можно записать в относительно простой форме 


$$
\begin{gathered}
\beta_{i+1 / 2}=4 \sqrt{a_{i} \Delta t}, \quad \beta_{i-1 / 2}=4 \sqrt{a_{i-1} \Delta t} \\
w\left(\xi_{i}, t^{n+1}\right)=-\frac{H\left(\xi_{i+1 / 2}, t\right) k\left(\xi_{i+1 / 2}, t_{n}\right) \alpha_{i+1 / 2}}{\beta_{i+1 / 2}}-\frac{H\left(\xi_{i-1 / 2}, t\right) k\left(\xi_{i-1 / 2}, t_{n}\right) \alpha_{i-1 / 2}}{\beta_{i-1 / 2}} \\
\operatorname{th}\left[\frac{\left(\xi_{i+1 / 2}-\xi_{i}\right) / 2}{\sqrt{a_{i} \Delta t}}\right]+\operatorname{th}\left[\frac{\left(\xi_{i}-\xi_{i-1 / 2}\right) / 2}{\sqrt{a_{i-1} \Delta t}}\right]
\end{gathered}
$$

С учетом предельных ситуаций $(h / 2)^{2} / a_{i} \Delta t \rightarrow 0$ и $(h / 2)^{2} / a_{i} \Delta t \rightarrow \infty$, ограничитель $\alpha$ потока $w$ можно описать формулами

$$
\begin{gathered}
\alpha_{i+1 / 2}=\frac{1+\operatorname{sign}\left(h / 2-\sqrt{a_{i} \Delta t}\right)}{2}\left[\frac{1}{\sqrt{\pi}} \exp \left(-\frac{(h / 2)^{2}}{a_{i} \Delta t}\right)\right]+\frac{1-\operatorname{sign}\left(h / 2-\sqrt{a_{i} \Delta t}\right)}{2}, \\
\alpha_{i-1 / 2}=\frac{1+\operatorname{sign}\left(h / 2-\sqrt{a_{i-1} \Delta t}\right)}{2}\left[\frac{1}{\sqrt{\pi}} \exp \left(-\frac{(h / 2)^{2}}{a_{i-1} \Delta t}\right)\right]+\frac{1-\operatorname{sign}\left(h / 2-\sqrt{a_{i-1} \Delta t}\right)}{2}
\end{gathered}
$$

Для получения конечно разностного представления (в варианте с условием $\left.w_{i}^{n+1} \approx w_{i-1 / 2}^{n+1} \approx w_{i+1 / 2}^{n+1}\right)$ уравнения $\left(C(\xi, t) \frac{\partial H}{\partial t}=-\frac{\partial w}{\partial \xi}\right)$ дивергентного закона сохранения энергии проинтегрируем его на отрезке $\left[\xi_{i}, \xi_{i+1}\right]$

$$
w\left(\xi_{i+1}, t^{n+1}\right)-w\left(\xi_{i}, t^{n+1}\right)+\frac{\left(H_{i+1 / 2}^{n+1}-H_{i+1 / 2}^{n}\right.}{\Delta t} \int_{\xi_{i}}^{\xi_{i+1}} C(\xi, t) \mathrm{d} \xi=0
$$

Подставляя в данное балансовое соотношение, найденное выражение для потока $w\left(\xi_{i}, t^{n+1}\right)$, получим следующую трехточечную разностную схему:

$$
A_{i+1 / 2} H_{i-1 / 2}^{n+1}-C_{i+1 / 2} H_{i+1 / 2}^{n+1}+B_{i+1 / 2} H_{i+3 / 2}^{n+1}=-F_{i+1 / 2},
$$

где коэффициенты $A_{i+1 / 2}, C_{i+1 / 2}, B_{i+1 / 2}, F_{i+1 / 2}$ трехточечной разностной схемы задаются формулами

$$
\begin{aligned}
& B_{i+1 / 2}=\frac{k\left(\xi_{i+3 / 2}, t_{n}\right) \alpha_{i+3 / 2} / \beta_{i+3 / 2}}{\operatorname{th}\left[\frac{\left(\xi_{i+3 / 2}-\xi_{i+1}\right) / 2}{\sqrt{a_{i+1} \Delta t}}\right]+\operatorname{th}\left[\frac{\left(\xi_{i+1 / 2}-\xi_{i}\right) / 2}{\sqrt{a_{i} \Delta t}}\right]}, \\
& A_{i+1 / 2}=\frac{k\left(\xi_{i-1 / 2}, t_{n}\right) \alpha_{i-1 / 2} / \beta_{i-1 / 2}}{\operatorname{th}\left[\frac{\left(\xi_{i+1 / 2}-\xi_{i}\right) / 2}{\sqrt{a_{i} \Delta t}}\right]+\operatorname{th}\left[\frac{\left(\xi_{i}-\xi_{i-1 / 2}\right) / 2}{\sqrt{a_{i-1} \Delta t}}\right]} \text {, }
\end{aligned}
$$

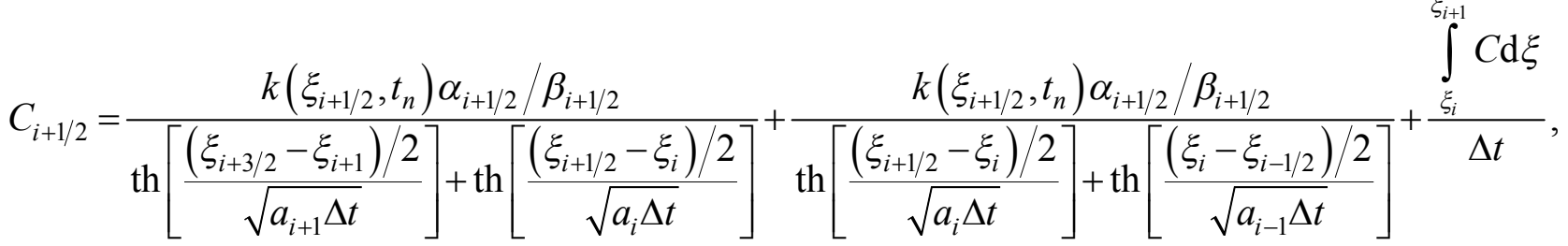




$$
F_{i+1 / 2}=\frac{H_{i+1 / 2}^{n} \int_{\xi_{i}}^{\xi_{i+1}} C \mathrm{~d} \xi}{\Delta t}
$$

Уравнения диффузии магнитного поля или уравнение теплопроводности относительно производной по времени представляют собой вариант системы ОДУ 1-го порядка. Эти уравнения можно проинтегрировать со вторым порядком точности с помощью двухстадийного монотонного варианта метода Рунге - Кутта

$$
\begin{gathered}
H^{(\mathrm{n}+1)^{*}}=H^{(\mathrm{n})}+\Delta t R\left(H^{(\mathrm{n})}\right), \quad R\left(w^{n+1}\right)=-\frac{1}{C\left(\xi_{i+1 / 2}, t_{n}\right)} \frac{\partial w^{n+1}}{\partial \xi}, \\
H^{(\mathrm{n}+1)}=\frac{1}{2} H^{(\mathrm{n})}+\frac{1}{2}\left[H^{(\mathrm{n}+1)^{*}}+\Delta t R\left(H^{(\mathrm{n}+1)^{*}}\right)\right]
\end{gathered}
$$

где $R$ - правая часть системы дифференциальных уравнений.

Далее аппроксимированное значение первой производной будем определять с использованием последовательности сеток (относительно временной переменной $t$ ) и экстраполяции Ричардсона [16]. Напомним, что перенос широкополосного излучения в одномерных задачах МИТС может рассматриваться на основе многогруппового диффузионного приближения (система уравнений данного приближения описана выше по тексту) [5].

\section{4. Результаты расчета}

При описании полученных численных результатов использовано обозначение: $N_{f u s}=\int_{0}^{t} \int_{0}^{l} Q_{F u s}^{e}(r, t) \times 3.567 \times 10^{4} \times 2 \pi r \mathrm{~d} r \mathrm{~d} t \quad[\mathrm{H} / \mathrm{cm}]-$ число нейтронов (на единицу длины мишени МИТС), покинувших расчетную область к моменту времени $t$.

Расчетная область и мишень МИТС состоят из центральной части и одного коаксиального слоя:

- центральная часть мишени (радиус центральной части $R_{\mathrm{L}}=0.05 \mathrm{~cm}$ ) заполнена D-T смесью с плотностью $\rho=5 \times 10^{-2} \Gamma / \mathrm{cm}^{3}$ и начальной температурой равной $T=297 \mathrm{~K}$.

Она окружена коаксиальным слоем (наружный радиус $R_{c}=0.1 \mathrm{~cm}$ ), состоящим из «твердого» D с плотностью $\rho=2.7 \times 10^{-2} \Gamma / \mathrm{cm}^{3}$ и начальной температурой равной $T=297 \mathrm{~K}$.

- $\quad$ расчетная область имеет внешний радиус $l=0.2$ см.

Начальное значения напряженности «затравочного» магнитного поля в окружающей среде составляет доли Тл. Спектральный поток и объемная плотность широкополосного излучения для $r \in[0, \ell]$ в начальный момент времени $t=0$ равны нулю.

Введем дополнительные обозначения необходимые для корректного обсуждения полученных численных результатов. Известно [34], что интенсивность ударной волны можно характеризовать безразмерной величиной, называемой амплитудой УВ: $Z=\left(P_{2}-P_{1}\right) / \rho_{1} c_{1}^{2}$, где индексы 1, 2 соответствуют газодинамическим параметрам плазмы до и после фронта УВ. Кроме этого известно, что наиболее существенной особенностью процесса взаимодействия УВ с геометрической осью мишени МИТС является усиление УВ после её отражения [34].

Процесс отражения УВ от оси мишени МИТС удобно характеризовать соответствующим коэффициентом усиления 


$$
K=\frac{\Delta P_{3}}{\Delta P_{2}},
$$

где $\Delta P_{3}=P_{3}-P_{1}-$ избыточное давление в отраженной УВ; $\Delta P_{2}=P_{2}-P_{1}-$ избыточное давление в падающей УВ.

Скорость движения ударной волны $V_{D}$ сквозь материал мишени можно в первом приближении оценить через скорость звука $V_{D} \approx \sqrt{\gamma R_{0} T / \mu}$, а скорость волны испарения $V_{s}$ следует из приближенного выражения $V_{s}(t) \approx L_{v} \rho / q_{l a z}$ или формулы работы [35], где $L_{v}-$ теплота сублимации материала стенки.

Скорость движения фронта «тепловой» волны $V_{f}$ определяется с помощью соотношения [36]

$$
V_{f}=\frac{r_{f}}{\tau(3 m+2)}
$$

где $r_{f}=\sqrt{\chi \tau} ; \tau-$ время действия лазерного импульса; $\chi=\frac{16 A \sigma}{3 \rho C_{v}} T^{m}=a T^{m}-$ коэффициент лучистой температуропроводности [36]; $\sigma=5.67 \times 10^{-8} \mathrm{BT} / \mathrm{M}^{2} \mathrm{~K}^{4}-$ постоянная СтефанаБольцмана; $A$ - величина обратная коэффициенту поглощения вещества; $C_{v}$ - теплоемкость сплошной среды.

Из результатов работ [13, 37-39] и приведенных в работе расчетов следует, что «сжатие» мишени МИТС возможно в нескольких режимах. При относительно невысоких значениях плотности потока лазерного импульса $\left.q_{l a z}(t)\right|_{r=\ell}$ через стенку мишени распространяется ударная волна (при условии, что внешнее давление превышает тепловое давление материала стенки мишени МИТС). При увеличении интенсивности плотности потока лазерного импульса $\left.q_{\text {laz }}(t)\right|_{r=\ell}$ на наружной поверхности мишени формируется волна испарения, толкающая мишень к оси симметрии. При этом перед стенкой формируется УВ, которая распространяется по центральной части мишени в направлении её оси симметрии. Если продолжать увеличение интенсивности плотности потока лазерного импульса $\left.q_{l a z}(t)\right|_{r=\ell}$, то в материале стенки мишени возникает «тепловая» волна, которая перемещает только энергию (но не массу - стенку мишени МИТС) и «сжатие» мишени заметно ослабляется.

При этом процесс комбинированного сжатия, относительно времени $t$, можно условно разделить на три характерные стадии, в пределах которых реализуются указанные режимы «сжатия» мишени МИТС:

- $\quad$ «начальная» стадия сжатия мишени МИТС в первой серии ударных волн;

- стадия сжатия мишени МИТС при «обострении» временного импульса;

- $\quad$ стадия ограничения расширения плазменного образования внешней импульсной системой струй.

Так из фазы «расширения» (момент времени $t=9.99$ нс) следует, что максимальное значение температуры $T=17.5 \times 10^{3} \mathrm{kK}$, статического давления $P=7 \times 10^{7}$ атм и плотности $\rho=80$ кг $/ \mathrm{M}^{3}$ наблюдается в интервале координат $0.015 \leq r \leq 0.03$ см. Границей плазменного образования является ударная волна со следующими характеристиками: $T \approx 17.1 \times 10^{3} \mathrm{kK}$, $P \approx 2.5 \times 10^{7}$ атм, $\rho \approx 1.6 \times 10^{2}$ кг $/ \mathrm{M}^{3}$, амплитуда УВ равна $Z \approx 10^{6}$. Магнитное давление $P_{m a g}$ к данному моменту времени увеличилось и имеет значение $P_{m a g} \approx 1.7 \times 10^{5}$ атм, число нейтронов (на единицу длины мишени МИТС), покинувших расчетную область максимально и равно $N_{\text {fus }}=3.08 \times 10^{14} \mathrm{H} / \mathrm{cm}$.

С момента времени $t_{0}<t$ (в пределах интервала $t_{0}<t<t_{0}+\Delta t$, на фазе «расширения» центральной части мишени, рис. 1-3) поток лазерного излучения с интенсивностью 
$q_{l a z}^{1}=1 \times 10^{15}\left[\mathrm{BT} / \mathrm{cm}^{2}\right]$, поступает также в пространственную область $0<r \leq 0.08$ см (через торцевую часть цилиндрической мишени МИТС. Энергия, подведенная к плазме, которая сформирована в течении трех стадий «сжатия» мишени МИТС, к центральной части мишени, за время $t=(10 \div 10.2)$ нс приводит к увеличению (в интервале координат $0.015 \leq r \leq 0.03 \mathrm{~cm})$ плазмодинамических параметров (с $T=17.5 \times 10^{3} \mathrm{kK}$ до $T=40 \times 10^{3} \mathrm{kK}$; с $P=3 \times 10^{8}$ атм до $P=2.6 \times 10^{9}$ атм) в области их максимальных значений. В результате процесса нагрева и интенсивного расширения формируется УВ, скорость движения ( $u \approx 250$ км/c) которой направлена в сторону оси симметрии мишени МИТС.

Перед УВ (вблизи оси симметрии) возникает «тепловая волна», которая движется в туже сторону, что и УВ. В момент времени $t=10.27$ нс (рис. 1) эти волны кумулируют (с интенсивным протеканием ядерных реакций) на оси симметрии. Этот процесс взаимодействия УВ с осью симметрии сопровождается существенным усилением (за счет протекания термоядерных реакций) отраженных волн (УВ и «тепловой», с ростом температуры плазмы до уровня $T=250 \times 10^{3} \mathrm{kK}, P=2.6 \times 10^{9}$ атм). В последующий момент времени «тепловая волна» распространяется до передней УВ $(r \approx 0.08$ см). Графическое представление, описанного выше процесса, приведено на рис. 1 и рис. 2.
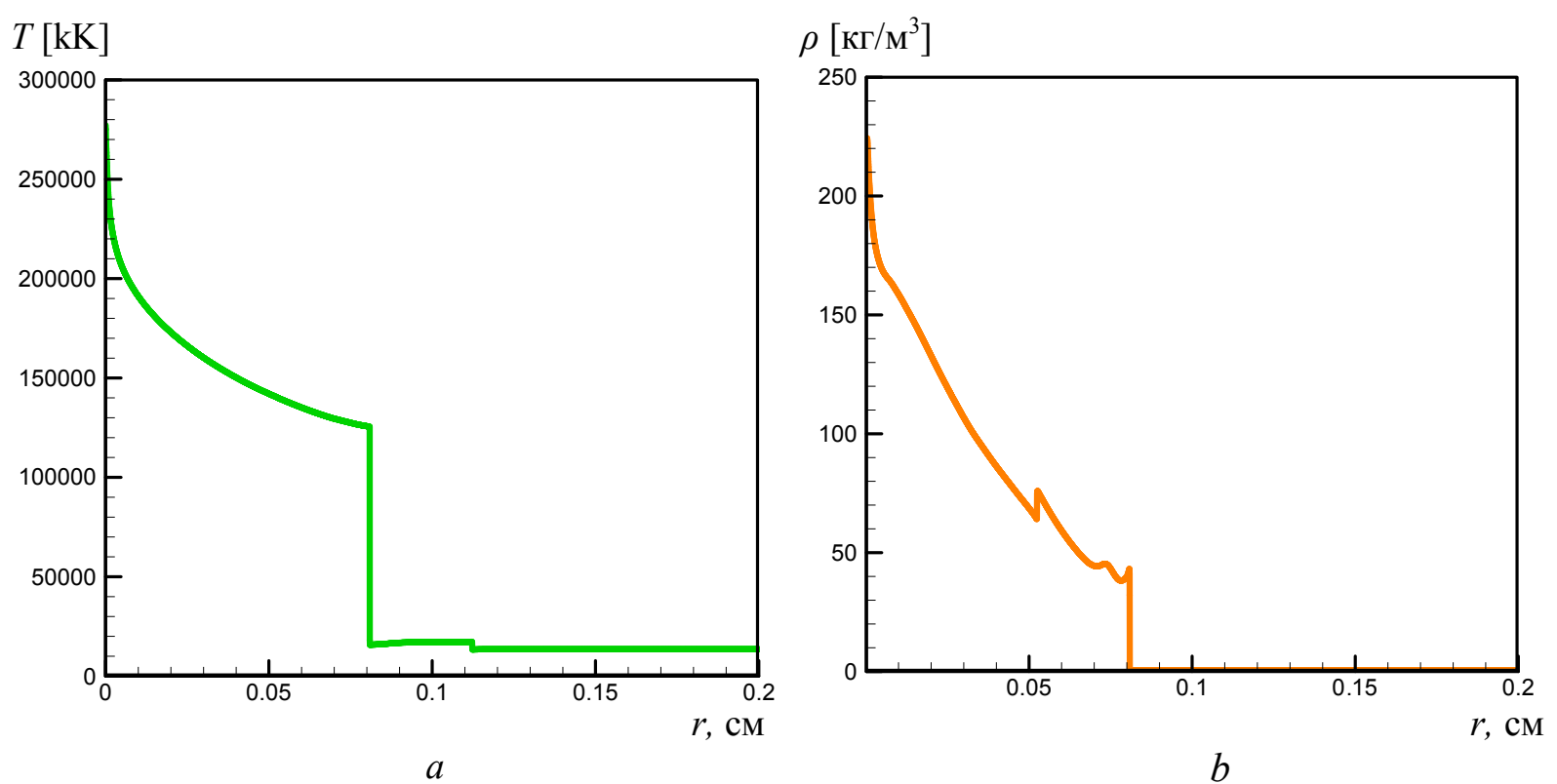

Рис. 1. Пространственное распределение температуры $T[\mathrm{kK}](a)$ и плотности $\rho\left[\kappa г / \mathrm{M}^{3}\right](b)$ для $q_{\text {laz }}^{0}=1 \times 10^{14}\left[\mathrm{BT} / \mathrm{cm}^{2}\right] \quad$ и $q_{\text {laz }}^{1}=1 \times 10^{15}\left[\mathrm{BT} / \mathrm{cm}^{2}\right]$ число нейтронов на единицу длины $N_{\text {fus }}=1.03 \times 10^{15} \mathrm{H} / \mathrm{cm}$ для момента времени $t=10.3 \mathrm{Hc}$

Главным физическим фактором, ответственным за рост параметров плазмы (рис.1) вблизи оси симметрии мишени МИТС, является увеличение интенсивности ударной волны, падающей на геометрическую ось системы.

Для объяснения указанного явления примем за меру интенсивности ударной волны число Маха М (иногда, называемое числом Маха УВ): $\mathrm{M}_{1}=\left(u_{1}-D\right) / c_{1}, \mathrm{M}=D / c_{1}$, где $u_{1}-$ скорость воздуха перед фронтом УВ, $D=\mathrm{M} \cdot c_{1}$ - скорость фронта УВ, $c_{1}, \rho_{1}, P_{1}$ - скорость звука, плотность и давление перед фронтом УВ. Тогда можно определить (через число Маха М и параметры $c_{1}, \rho_{1}, P_{1}$ перед УВ) плотность $\rho_{2}$, скорость $u_{2}$ и давление $P_{2}$ за фронтом УВ с помощью соотношений

$$
\frac{\rho_{2}}{\rho_{1}}=\frac{(\gamma+1) \mathrm{M}^{2}}{(\gamma-1) \mathrm{M}^{2}+2}, \quad u_{2}=\mathrm{M} \cdot c_{1}\left(1-\frac{\rho_{1}}{\rho_{2}}\right), \quad \frac{P_{2}}{P_{1}}=\frac{2 \gamma \mathrm{M}^{2}}{\gamma+1}-\frac{\gamma-1}{\gamma+1}
$$


Из этих соотношений можно сделать вывод, что выражения $\rho_{2} / \rho_{1}=f_{1}(\mathrm{M}, \gamma)$, $P_{2} / P_{1}=f_{2}(\mathrm{M}, \gamma), u_{2} \approx f_{3}\left(\mathrm{M}, c_{1}\right)$ являются функциями числа Маха $\mathrm{M}=D / c_{1} \approx\left(u_{2}+c_{2}\right) / c_{1}$.
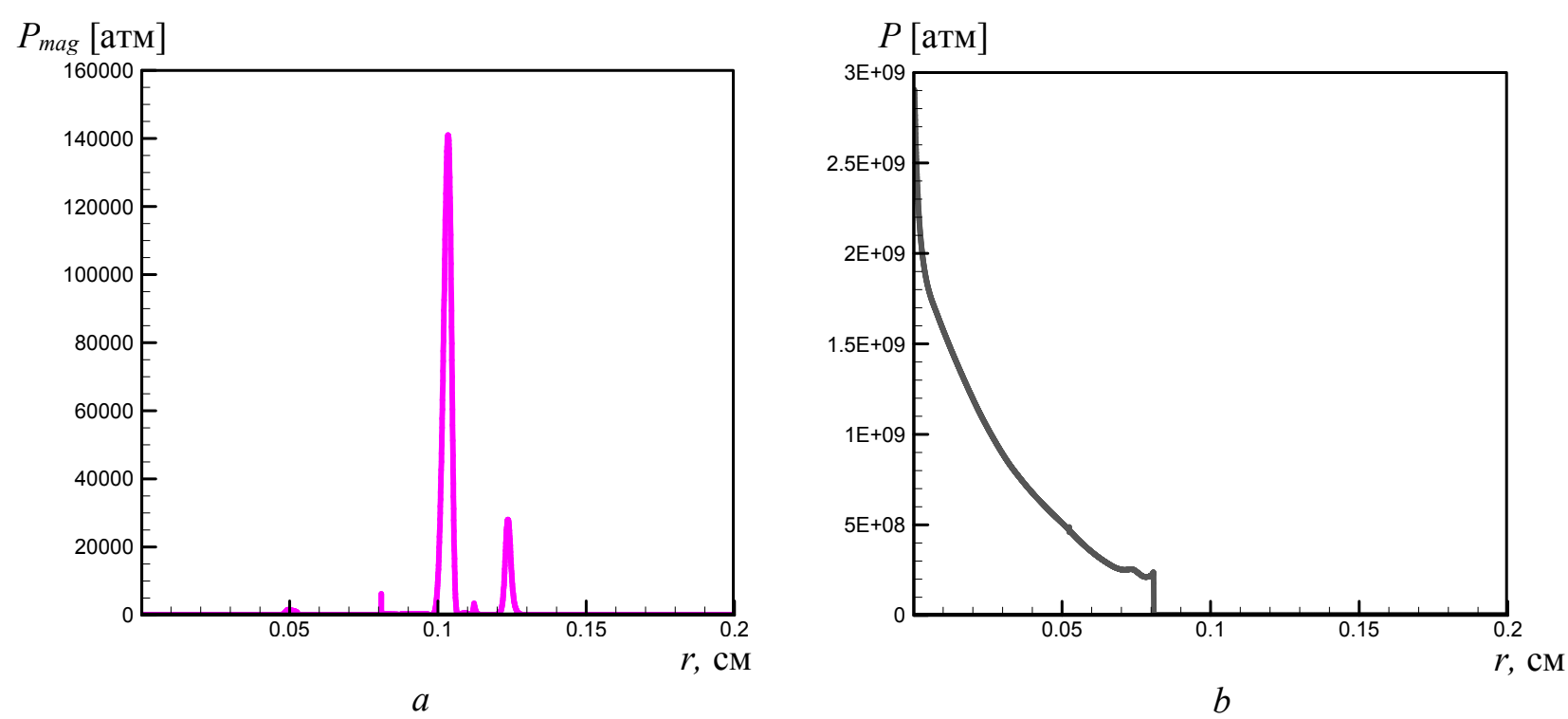

Рис. 2. Пространственное распределение магнитного давления $P_{m a g}$ [атм] $(a)$ и статического давления для $q_{l a z}^{0}=1 \times 10^{14}\left[\mathrm{BT} / \mathrm{cm}^{2}\right]$ и $q_{l a z}^{1}=1 \times 10^{15}\left[\mathrm{BT} / \mathrm{cm}^{2}\right]$ число нейтронов на единицу длины $N_{\text {fus }}=1.03 \times 10^{15} \mathrm{H} / \mathrm{cm}$ для момента времени $t=10.3 \mathrm{Hc}$

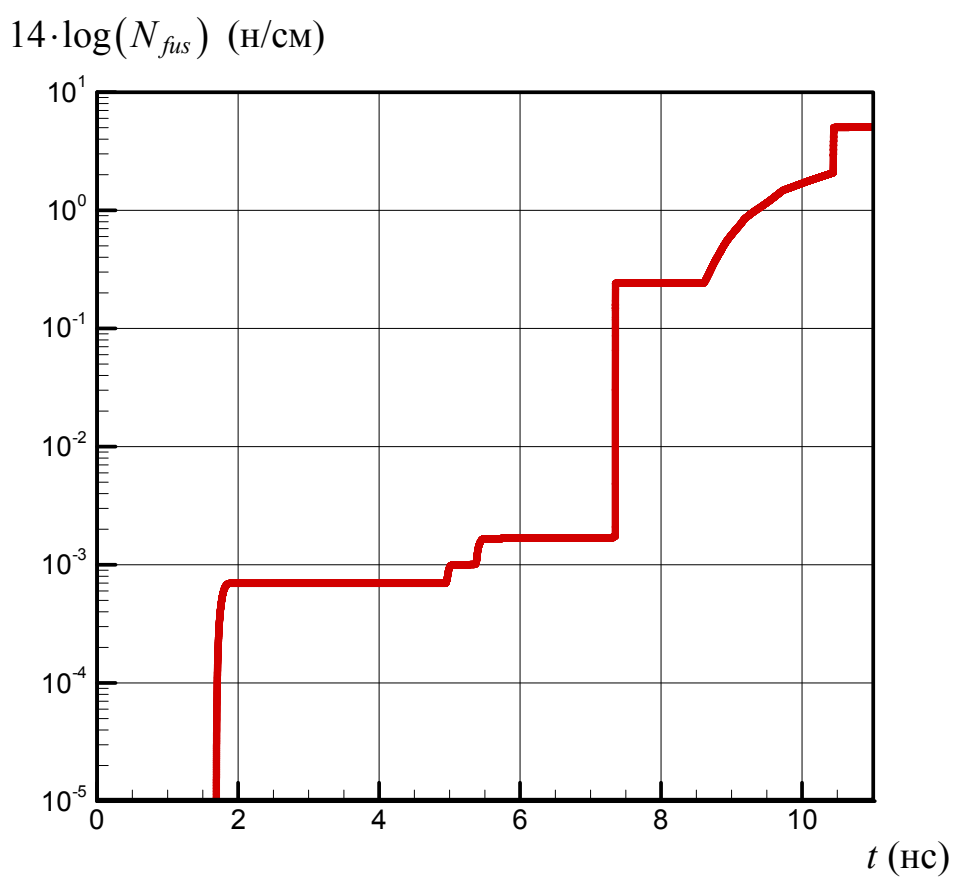

Рис. 3. Число нейтронов $N_{f u s}$ н/см для $q_{\text {laz }}^{0}=1 \times 10^{14}\left[\mathrm{BT} / \mathrm{cm}^{2}\right]$ и $q_{l a z}^{1}=5 \times 10^{15}\left[\mathrm{BT} / \mathrm{cm}^{2}\right]$, покинувших расчетную область к моменту времени $t$

Отсюда следует, что интенсивность падающей на ось мишени УВ может быть заметно повышена в том случае, когда отношение скоростей звука $c_{2}\left(T_{2}\right) / c_{1}\left(T_{1}\right)$ достаточно велико $\left(c_{2} / c_{1} \gg 1, \mathrm{M} \gg 1\right.$; при этом $\left.P_{2} / P_{1} \approx 2 \gamma \mathrm{M}^{2} /(\gamma+1) \gg 1\right)$. Этого увеличения можно добиться с помощью повышения температуры $T_{2}$ за фронтом УВ на завершающей стадии сжатия (например, путем подвода энергии лазерным излучением через торец мишени МИТС к ударной сжатой плазме, за фронт падающей на ось симметрии УВ). 


\section{5. Заключение}

В работе дана оценка плазмодинамических параметров однослойной цилиндрической мишени МИТС для комбинированной схемы воздействия интенсивных потоков энергии. В этой схеме осуществляется последовательное воздействие интенсивного лазерного излучения (с максимальной плотностью потока излучения $q_{l a z}^{0}=10^{14} \mathrm{BT} / \mathrm{cm}^{2}$ ) вдоль образующей однослойной цилиндрической мишени (до момента времени $t \leq t_{0}$ ), затем с момента времени $t_{0}<t$ внешней (по отношению к мишени) системы импульсных струй (с плотностью потока энергии $10^{12} \mathrm{BT} / \mathrm{cm}^{2}$ ) и лазерного излучения (в торцевом направлении) с интенсивностью $q_{l a z}^{1}=1 \times 10^{15} \mathrm{Bт} / \mathrm{cm}^{2}$.

В работе приведен новый вариант метода численного решения одномерных уравнений плазмодинамики. Этот метод основан (для «гиперболической» части системы уравнений (1)-(7)) на нелинейной квазимонотонной компактно-полиномиальной разностной схеме повышенного порядка точности, а для «параболической» части системы уравнений на новом (он позволяет выполнять расчеты в случаях интенсивных разрывов в транспортных коэффициентах) численном методе решения уравнения диффузии магнитного поля и уравнения теплопроводности с помощью монотонизированной разностной схемы повышенного порядка точности. Разработанная численная методика была верифицирована с помощью решения ряда тестовых (модельных) задач [43, 42]. «Гиперболическая» (конвективная) части компьютерной модели мишеней МИТС тестировалась на одномерном варианте задачи Римана (задача Сода) о распаде неустойчивого разрыва заданной конфигурации. Сопоставление точного и приближенного решения показало, что отличие составляет не более процента $[42,44]$. Верификационные расчеты были проведены с целью оценки степени затухания системы отраженных ударных волн и показали, что ошибка расчета находится в пределах экспериментальной точности результатов и может достигать уровня $10 \%$. В качестве дополнительных тестовых верификационных расчетов рассматривается обтекание потоком воздухом клина, сопряженного с пластиной, а также конуса, сопряженного с цилиндром со следующими параметрами набегающего потока: давление $P=2060$ Па, скорость $V=1860$ м/с, температура $T=223 \mathrm{~K}$, число Маха $M_{\infty}=6$. Данные результаты также находится в хорошем соответствии [42] с указанными расчетами (относительная ошибка равна $0.4 \%$ ). «Тепловая» («параболическая») часть модели тестировалась на некоторых задачах, допускающих точные аналитические решения: прогрев сплошной среды [43, 44], заполняющей плоское полуограниченное пространство $r>0$, потоком тепла, через левую неподвижную границу $r=0$ (относительная ошибка меньше $1.0 \%$ ).

В работе проанализированы все стадии сжатия мишени МИТС для комбинированной схемы воздействия и показано, что температура центральной части мишени может достигать в отдельные моменты времени уровня $T=250$ млн.К. С помощью выполненных расчетов дана оценка величины магнитного поля, индуцируемого сжатием мишени. Показана (рис.3) возможность создания генераторов нейтронов на основе комбинированной схемы воздействия (число нейтронов на единицу длины $N_{f u s}=1.03 \times 10^{15} \mathrm{H} / \mathrm{cm} \mathrm{к} \mathrm{моменту} \mathrm{времени}$ окончания воздействия). Здесь отметим, что независимо от выбранного типа мишени МИТС в процессе сжатия могут развиваться гидродинамические неустойчивости, препятствующие достижению оптимальных параметров термоядерного горючего.

В данной работе не учитывается влияние несимметрии при расчете сжатия мишени МИТС, что может приводить к различию в наблюдаемом выходе нейтронов (в два порядка) от предсказаний одномерных расчетов. Известно, что независимо от выбранного типа мишени МИТС в процессе сжатия могут развиваться гидродинамические неустойчивости, препятствующие достижению оптимальных параметров термоядерного горючего. Как показано [40], развитие неустойчивости происходит, когда градиенты давления и плотности противоположно направлены, т.е. $\nabla P \cdot \nabla \rho<0$. В основном это имеет место на двух стадиях [41] сжатия мишени: 
1. на внешней поверхности оболочки мишени во время её ускорения малоплотным абляционным слоем - так называемая абляционная неустойчивость;

2. на внутренней поверхности оболочки мишени во время её торможения горячим центром, находящимся под высоким давлением.

Bсе эти неустойчивости является следствием несимметричного характера лазерного облучения. А это в свою очередь приводит к несимметричному расположению топлива (в процессе сжатия) относительно центра мишени и, следовательно, к снижению температуры, плотности горючего и значительному (в два порядка) снижению нейтронного выхода отдельных (с разными температурами, плотностями и скоростями) областей мишени МИТС. При этом мелкомасштабные возмущения формы поверхности мишени с начальной амплитудой в несколько микрон слабо влияют на процесс сжатия мишени, а снижение нейтронного выхода составляет не более 2-3 раз (нейтронный выход может уменьшаться при увеличении ширины зоны перемешивания) по сравнению с симметричным сжатием.

Выполненные в работе 1D расчеты плазмодинамических параметров цилиндрической мишени МИТС для комбинированной схемы воздействия, учитывают широкий спектр физических эффектов и в целом дают информацию о динамике процессов в тех участках 3D мишени, где плотность изучения лазера либо наиболее интенсивна, либо относительной мала. То есть 1D расчеты в принципе (без учета взаимного влияния) позволяют получить картины 2D распределений поглощенной энергии, плазмодинамических и термодинамических величин, а значить и судить о работоспособности комбинированной схемы воздействия на мишень МИТС.

Из сказанного также следует, что учет не одномерности физических процессов и вопросов, связанных с гидродинамическими неустойчивостями (возникающие в процессе сжатия мишени МИТС), из которых основную роль играют неустойчивости Рэлея-Тейлора и Рихтмайера-Мешкова, требует дальнейшего отдельного, подробного изучения.

\section{Благодарности и ссылки на гранты}

Результаты исследования получены в рамках выполнения государственного задания Министерства науки и высшего образования Российской Федерации (Минобрнауки России) № 13.5240.2017/8.9 и поддержаны Целевой программой МГТУ им. Н.Э. Баумана на 20182020 годы.

\section{Литература}

1. Митчнер М., Кругер Ч. Частично ионизованные газы - М.: Мир, 1976, 496 с.

2. Брагинский С.И. Явления переноса в плазме - В книге: Вопросы теории плазмы. Вып.1 М.: Атомиздат, 1963, с. 183-272

3. Афанасьев Ю.В., Гамалий Е.Г., Розанов В.Б. Основные уравнения динамики и кинетики лазерной плазмы. Труды ФИАН АН СССР. 1982. Т. 134. С. 10-31.

4. Бретшнайдер С. Свойства газов и жидкостей. М.: Химия, 1966. 536 с.

5. Четверушкин Б.Н. Математическое моделирование задач динамики излучающего газа. - М.: Наука, 1985. - 303 с.

6. Кузенов В.В. Компьютерное моделирование процесса сжатия и энерговыделения при лазерном воздействии на цилиндрически симметричную мишень // Ядерная физика и инжиниринг. 2016. T. 7, № 4. C. 342-346.

7. Kuzenov V.V., Ryzhkov S.V. Evaluation of hydrodynamic instabilities in inertial confinement fusion target in a magnetic field // Problems of Atomic Science and Technology. 2013. № 4 (86). Pp. 103-107. 
8. Ryzhkov S.V., Chirkov A.Yu., Ivanov A.A. Analysis of the compression and heating of magnetized plasma targets for magneto-inertial fusion // Fusion Science and Technology. 2013. V. 63. № 1T. Pp. 135-138.

9. Козлов Б.Н. Скорости термоядерных реакций // Атомная энергия, 1962, т. 12, № 3, с. 238.

10. Самарский А.А., Попов Ю.П. Разностные методы решения задач газовой динамики. - М.: Едиториал УРСС, 2009. 424 с.

11. Кузенов В.В., Лебо А.И., Лебо И.Г., Рыжков С.В. Физико-математические модели и методы расчета воздействия мощных лазерных и плазменных импульсов на конденсированные и газовые среды (2-е изд.). М.: МГТУ им. Н.Э. Баумана, 2017. 328 с.

12. Kuzenov V.V., Ryzhkov S.V. Numerical simulation of the effect of laser radiation on matter in an external magnetic field // Journal of Physics: Conference Series. 2017. V. 830. P. 012124.

13. Кузенов В.В., Рыжков С.В. Численное моделирование процесса лазерного сжатия мишени, находящейся во внешнем магнитном поле // Математическое моделирование. 2017. Т. 29. № 9. C. 19-32.

14. Крайко А. Н. Теоретическая газовая динамика (краткий курс). - М.: МФТИ, 2007. -300 с.

15. Волосевич П.П., Дегтярев Л.М., Леванов Е.И. и др. Процесс сверхвысокого сжатия вещества и инициирования термоядерной реакции мощным импульсом лазерного излучения // Физика плазмы, т.2, вып.6, 1975, С. 883-897.

16. Kuzenov V.V., Ryzhkov S.V. Calculation of plasma dynamic parameters of the magneto-inertial fusion target with combined exposure // Physics of Plasmas. 2019. V. 26. P. 092704.

17. Surzhikov S.T. Computing system for solving radiative gasdynamic problems of entry and re-entry space vehicles // Proceedings of the 1st International Workshop on Radiation of High Temperature Gases in Atmospheric Entry, 2003, ESA-533, pp. 111-118.

18. Кузенов В.В., Рыжков С.В., Шумаев В.В. Определение термодинамических свойств замагниченной плазмы на основе модели Томаса-Ферми // Прикладная физика. 2014. № 3. С. 22-25.

19. Kuzenov V.V., Ryzhkov S.V., Shumaev V.V. Application of Thomas-Fermi model to evaluation of thermodynamic properties of magnetized plasma // Problems of Atomic Science and Technology. 2015. No. 1 (95). Pp. 97-99.

20. Ковеня В.М., Яненко Н.Н. Метод расщепления в задачах газовой динамики. - М.: Наука, 1981. $-304 \mathrm{c}$.

21. Волков К.Н., Емельянов В.Н. Моделирование крупных вихрей в расчетах турбулентных течений. - М.: Физматлит, 2008. 364 с.

22. Марчук Г.И., Шайдуров В.В. Повышение точности решения разностных схем. - М.: Наука, 1979. - $320 \mathrm{c}$.

23. Довгилович Л.Е., Софронов И.Л. О применении компактных схем для решения волнового уравнения, Москва, Препринт ИПМ АН СССР № 84, С. 1-27.

24. Barth T.J. On instructed grids and solvers, in Computational Fluid Dynamics (Von Karman Institute for Fluid Dynamics. 1990), Lecture Series 1990-04

25. Савельев А.Д. Составные компактные схемы высокого порядка для моделирования течения вязкого газа. // ЖВМ и МФ, - 2007. - Т.47, №8 - С. 1387-1401.

26. Z. Xu and C.-W. Shu, Anti-diffusive high order WENO schemes for Hamilton-Jacobi equations, Method and Application of Analysis, 12 (2005), pp. 169-190.

27. Z. Xu and C.-W. Shu, Anti-diffusive flux corrections for high order finite difference WENO schemes, Journal of Computational Physics, 205 (2005), pp. 458-485.

28. Kuzenov V.V., Ryzhkov S.V., Shumaev V.V. Numerical thermodynamic analysis of alloys for plasma electronics and advanced technologies // Problems of Atomic Science and Technology. 2015. No. 4 (98). Pp. 53-56.

29. Пинчуков В.И., Шу Ч.В. Численные методы высоких порядков для задач аэрогидродинамики. Новосибирск: Изд-во Сиб. отд. РАН, 2000. 
30. Абрамовиц М., Стиган И. Справочник по специальным функциям. М.: Наука, 1979.

31. Лаврентьев М.А., Шабат Б.В. Методы теории функций комплексного переменного. М.: Наука, 1973.

32. ВорожцовЕ.В. "Применение разложений Лагранжа-Бюрмана для численного интегрирования уравнений невязкого газа", Выч. мет. программирование, 12:3 (2011), pp. 348-361.

33. Ryzhkov S.V., Kuzenov V.V. Analysis of the ideal gas flow over body of basic geometrical shape // International Journal of Heat and Mass Transfer. 2019. V. 132. Pp. 587-592.

34. Овсянников Л.В. Лекции по основам газовой динамики. М.: Наука, 1981. - 368 с.

35. Лебо И.Г., Тишкин В.Ф. Исследование гидродинамической неустойчивости в задачах лазерного термоядерного синтеза методами математического моделирования М. Физматлит, 2006 г. $304 \mathrm{c}$.

36. Зельдович Я.Б., Райзер Ю.П. Физика ударных волн и высокотемпературных явлений гидродинамических явлений. М.: Наука, 1966.

37. Kuzenov V.V., Ryzhkov S.V. Numerical modeling of magnetized plasma compressed by the laser beams and plasma jets // Problems of Atomic Science and Technology. 2013. № 1 (83). Pp. 12-14.

38. Котов М.А., Рулева Л.Б., Солодовников С., Суржиков С.Т. Проведение экспериментов по обтеканию моделей в гиперзвуковой ударной аэродинамической трубе//Физико-химическая кинетика в газовой динамике. 2013. Т. 14, вып. 4. http://chemphys.edu.ru/issues/2013-144/articles/428/

39. Глушко Г.С., Иванов И.Э., Крюков И.А. Моделирование турбулентности в сверхзвуковых струйных течениях // Физико-химическая кинетика в газовой динамике. 2010. Том 9. http://chemphys.edu.ru/issues/2010-9/articles/142/

40. Фрадкин Е.С. Исследование устойчивости произвольного одномерного гидродинамического течения Труды Физического института им. П. Н. Лебедева. 1965. Т. 29. С. 250-256.

41. Афанасьев Ю.В., Басов Н.Г., Гамалий Е.Г. и др. Симметрия и устойчивость сжатия лазерных термоядерных мишеней Письма в ЖЭТФ. 1976. июнь. Т. 23, № 11. С. 617-620.

42. Kuzenov V.V., Ryzhkov S.V. Approximate method for calculating convective heat flux on the surface of bodies of simple geometric shapes // Journal of Physics: Conference Series. 2017. V. 815. P. 012024.

43. Mazhukin V.I., Malaphei D.A. et al., Comput. Mathem. and Mathem. Phys. 41, 2001. Pp. 379-391.

44. Kuzenov V.V., Ryzhkov S.V., Frolko P.A. Numerical simulation of the coaxial magneto-plasma accelerator and non-axisymmetric radio frequency discharge // Journal of Physics: Conference Series. 2017. V. 830. P. 012049. 